\title{
Subnormal operators of finite type II. Structure theorems
}

\author{
Dmitry V. Yakubovich
}

\begin{abstract}
This paper concerns pure subnormal operators with finite rank self-commutator, which we call subnormal operators of finite type. We analyze Xia's theory of these operators [21]-[23] and give its alternative exposition. Our exposition is based on the explicit use of a certain algebraic curve in $\mathbb{C}^{2}$, which we call the discriminant curve of a subnormal operator, and the approach of dual analytic similarity models of [26]. We give a complete structure result for subnormal operators of finite type, which corrects and strenghtens the formulation that Xia gave in [23]. Xia claimed that each subnormal operator of finite type is unitarily equivalent to the operator of multiplication by $z$ on a weighted vector $H^{2}$-space over a "quadrature Riemann surface" (with a finite rank perturbation of the norm). We explain how this formulation can be corrected and show that, conversely, every "quadrature Riemann surface" gives rise to a family of subnormal operators. We prove that this family is parametrized by the so-called characters. As a departing point of our study, we formulate a kind of scattering scheme for normal operators, which includes Xia's model as a particular case.
\end{abstract}

\section{Introduction.}

This paper is devoted to an alternative exposition of some aspects of Xia's theory of subnormal operators from a different viewpoint. We make use of the results of [25] and the approach of [26] and give new 
results and new connections. The ideas of Xia are exploited much throughout the paper, but our exposition is independent.

We develop a scattering scheme for normal operators, whose particular case is Xia's model, explain the role of the discriminant curve and the involution on it, and prove a complete structure result, which gives a two-sided connection between subnormal operators of finite type and real algebraic curves of a certain class.

The structure theorem we obtain in this paper allows one to prove an interesting relationship between subnormal operators of finite type and a certain class of vector analytic Toeplitz operators. This relationship gives rise to a new characterization of quadrature domains. These results will be presented elsewhere.

Let $H$ be a Hilbert space and $\mathcal{L}(H)$ the space of bounded linear operators on $H$. An operator $S \in \mathcal{L}(H)$ is called subnormal if there is a Hilbert space $K, K \supset H$ and a normal operator $N \in \mathcal{L}(K)$ such that $N H \subset H$ and $S=N \mid H . S$ is called pure if it has no nonzero reducing subspace on which it is normal. We will say that $S$ is of finite type if it is pure and $\operatorname{rank}\left[S^{*}, S\right]<\infty$ (here $\left[S^{*}, S\right]=S^{*} S-S S^{*}$ ).

Let $S$ be pure subnormal, and put

$$
\begin{aligned}
& M=\text { clos Range }\left[S^{*}, S\right], \\
& C=\left[S^{*}, S\right] \mid M, \\
& \Lambda=\left(S^{*} \mid M\right)^{*} .
\end{aligned}
$$

Xia has shown in [21] that $M$ is invariant for $S^{*}$ and that the pair of operators $C, \Lambda$ on $M$ completely determines $S$ up to the unitary equivalence. Operators $C, \Lambda$ play an essential role in Xia's model. For the case of a subnormal operator of finite type, the set of matrix parameters $(C, \Lambda)$ has been described completely in [25]. The answer was formulated in terms of the algebraic curve

$$
\Delta=\left\{(z, w): \operatorname{det}\left(C-\left(w-\Lambda^{*}\right)(z-\Lambda)\right)=0\right\}
$$

If $C, \Lambda$ correspond to a subnormal operator $S$, then $\Delta$ is called the discriminant curve of $S$.

Here we define a certain class of algebraic curves in $\mathbb{C}^{2}$, which we call admissible separated curves. An algebraic curve $\Delta$ is in this class if it has a prescribed behavior at infinity and the real linear manifold $w=\bar{z}$ divides each of its irreducible components into two connected parts. For such curve $\Delta$, there is a canonical way to define its "halves" 
$\Delta_{+}, \Delta_{-}$. Let $\widehat{\Delta}$ be the blow-up of $\Delta$. Each connected component of $\widehat{\Delta}$ is a compact Riemann surface, obtained from an irreducible component of $\Delta$ by deleting its singular points and then adding a finite number of "ideal" points [11].

For any admissible separated algebraic curve $\widehat{\Delta}$ and a matrixvalued function $\Omega$ on $\partial \widehat{\Delta}_{+}$with integrable $\log \|\Omega\|, \log \left\|\Omega^{-1}\right\|$, we introduce the weighted Hardy class $H^{2}\left(\widehat{\Delta}_{+}, \Omega\right)$. We show that the operator of multiplication by the variable $z$ on $H^{2}\left(\widehat{\Delta}_{+}, \Omega\right)$ is subnormal of finite type and that its discriminant curve is $\Delta$. (We call such subnormal operators simple.) We deduce from this fact that an algebraic curve is the nondegenerate part of the discriminant curve of a subnormal operator of finite type if and only if this curve is admissible and separated.

The main structure result we get shows that any subnormal operator of finite type is obtained from a simple subnormal operator by "glueing" finitely many points of $\widehat{\Delta}_{+}$and then performing a finite rank perturbation of the Hilbert space structure. Conversely, any such procedure gives a subnormal operator of finite type.

A criterion for unitary equivalence of subnormal operators of finite type is given. Roughly speaking, it consists in equality of certain characters (homeomorphisms of fundamental groups of the components of $\widehat{\Delta}_{+}$into suitable groups of unitary matrices). This criterion generalizes a result by McCarthy and Yang [16], who considered the rationally cyclic case.

In order to understand better Xia's model, in sections 1-3 we introduce its generalization. It has an operator theory face and a complex analysis face, and we study them separately.

The operator theory part of the construction has the form of a scattering type scheme for normal operators. We say that a tuple $\left(N, K, H^{\prime}, H, M\right)$ is a scattering tuple if $K$ is a Hilbert space, $H^{\prime}, H, M$ are its subspaces, the operator $N: K \longrightarrow K$ is similar to a normal operator, a direct sum decomposition $K=H^{\prime} \dot{+} H$ holds, and $N H \subset H$, $N H^{\prime} \subset H^{\prime}+M, M \subset H, \operatorname{dim} M<\infty$. With each such tuple we associate the operator $S=N \mid H$.

To formalize the complex analysis context, we introduce what we call mosaic tuples. A mosaic tuple consists of three matrix-valued functions and a scalar measure, interrelated in a certain way. Each mosaic tuple gives rise to a projection-valued mosaic $\mu$ and serves as a prerequisite for defining functional model spaces, which consist of analytic and antianalytic $M$-valued functions on $\mathbb{C} \backslash \sigma(N)$. The conclusion of sections $1-3$ is that the two settings are equivalent: to each mosaic tu- 
ple corresponds a unique scattering tuple, and vice versa. In a sense, the mosaic tuple plays the role of the characteristic function in these constructions. The consideration of a generalized Xia's model has the advantage that one can understand well the freedom in choosing parameters of the mosaic tuple (see Section 5). The class of operators $S$ which one gets in this way is much more general than the class of subnormal operators of finite type. For instance, the essential spectrum of a subnormal operator of finite type always lies on an algebraic curve, whereas the essential spectrum of an operator of the type considered in sections 1-3 can be any reasonable finite union of piecewise $C^{1}$-smooth curves.

In sections $1-3$, the ideas and approach of dual bundle shift models [26] are used. The connection with dual bundle shift models is explained in Section 4. These models have been used in [26] for studying Toeplitz operators and in [27] to study hyponormal operators. The results of Section 4 are not used in the sequel.

Then we use the scattering scheme of sections 1-3 to study Xia's original model. We show how the properties $H^{\prime}=H^{\perp}, M=\left[S^{*}, S\right] H$, which distinguish it, are connected with the existence of the antianalytic involution on $\widehat{\Delta}$. One of the outcomes of our exposition is a concrete explicit construction of a subnormal $S$ of finite type from matrices $C$ and $\Lambda$, if it exists.

The proof of the structure results we give consists in two reductions (whose idea is due to Xia). First we replace the mosaic model space $E^{2}(\mu)$ of functions on $\mathbb{C} \backslash \sigma(N)$ by a space of cross-sections of a certain analytic bundle over $\widehat{\Delta}_{+}$. Then, after trivializing this bundle and characterizing the space of its cross-sections (Section 10), we obtain our main structure results in Section 12. Necessary facts about weighted vector Hardy spaces over Riemann surfaces, characters and related topics are given in Section 9.

The relationship between subnormal operators and separated algebraic curves is most clear from Lemma 11.4 and its proof. The reader who just wishes to get an idea of the subject can first look at this lemma.

It is worth noticing that subnormal operators of finite type turn out to be unexpectedly close to Toeplitz operators with rational and similar symbols, which were studied in [18], [24]. In particular, the results about spectral multiplicity [18] and invariant and hyperinvariant subspaces [24] extend to subnormal operators of finite type.

Algebraic curves and Hardy classes over these curves also have been 
used intensively in works of Alpay, Fedorov, Livšic, Vinnikov and others (see [3], [8], [9], [14]). It would be interesting to know a connection between subnormal operators and the subject of those works.

At the end of the paper, an index of mathematical notation is given.

\section{Mosaic tuples and mosaic model spaces.}

Suppose we are given a compactly supported positive Borel measure $\nu$ on the complex plane, a finite-dimensional Hilbert space $M$ and $\mathcal{L}(M)$-valued measurable functions $F, \mathcal{E}, G$ on $\mathbb{C}$ such that $\mathcal{E}=\mathcal{E}^{*} \geqslant 0$ $\nu$-almost everywhere. Put $\gamma=\operatorname{supp} \nu$,

$$
d e(\cdot)=\mathcal{E}(\cdot) d \nu(\cdot)
$$

and

$$
p(u)=F(u) \mathcal{E}(u) G(u) .
$$

Consider the space

$$
L^{2}(e)=\left\{f:\|f\|^{2}=\int\langle\mathcal{E}(u) f(u), f(u)\rangle d \nu(u)<\infty\right\}
$$

After factoring by the set of functions $f$ with $\|f\|^{2}=0, L^{2}(e)$ becomes a Hilbert space. Each element of $L^{2}(e)$ has a unique representative $f$ such that $f(\cdot) \in$ Range $\mathcal{E}(\cdot) \nu$-almost everywhere. Two functions $f, g$ are equal in $L^{2}(e)$ if and only if $\mathcal{E} f=\mathcal{E} g$.

In the setting of sections $1-3$, there will be no loss of generality if we assume that $\mathcal{E}$ is a projection-valued function and $F=F \mathcal{E}, G=\mathcal{E} G$. Then $L^{2}(e)$ is the direct integral of the spaces $\mathcal{E}(\cdot) M$. We choose the formally more general setting in order to include the original Xia's mosaic.

We make the following assumptions.

M1) The function

$$
\mu(z)=\int_{\gamma} \frac{p(u)}{u-z} d \nu(u), \quad z \in \mathbb{C} \backslash \gamma
$$

is projection-valued. 
M2) $F^{*}(\cdot) m \in L^{2}(e)$ and $G(\cdot) m \in L^{2}(e)$ for any $m \in M$. The operators $F(u)\left|\mathcal{E}(u) M, G^{*}(u)\right| \mathcal{E}(u) M$ are one-to-one for $\nu$-almost every $u$.

M3) The family of functions $(\cdot-\lambda)^{-1} G(\cdot) m, m \in M, \lambda \in \mathbb{C} \backslash \gamma$ and the family of functions $(\bar{\cdot}-\bar{\lambda})^{-1} F^{*}(\cdot) m, m \in M, \lambda \in \mathbb{C} \backslash \gamma$ are complete in $L^{2}(e)$.

The function $\mu$ is "piecewise analytic", that is, it is analytic on $\mathbb{C} \backslash \gamma$. We call it a generalized Xia's mosaic. Since $\mu(\infty)=0$, it follows that $\mu \equiv 0$ in the unbounded component of $\mathbb{C} \backslash \gamma$.

Define the Cauchy integral

$$
\mathcal{K} f(z)=\int_{\gamma} \frac{f(t)}{t-z} d \nu(t), \quad z \in \mathbb{C} \backslash \gamma .
$$

By (M2) and (M3), the map $f \longmapsto \mathcal{K} F \mathcal{E} f, f \in L^{2}(e)$ is one-to-one. Let $\mathcal{K} F \mathcal{E} L^{2}(e)=\left\{\mathcal{K} F \mathcal{E} f: f \in L^{2}(e)\right\}$ be the image of this map, with the norm inherited from $L^{2}(e)$. We need also the following assumption.

M4) The operator

$$
\left(P_{\mu} u\right)(z)=\mu(z) u(z), \quad z \in \mathbb{C} \backslash \gamma
$$

acts on $\mathcal{K} F \mathcal{E} L^{2}(e)$ and is bounded.

Definition. We say that $(M, F, \mathcal{E}, G, \nu, \mu)$ is a mosaic tuple if M1)M4) hold.

ExAmple. Let $M=\mathbb{C}^{1}, \nu=|d z|$ on $\partial \mathbb{D}$, where $\mathbb{D}=\{|z| \leqslant 1\}$, $p(z)=z / 2 \pi$, so that $\mu(z)=1$ for $|z|<1$ and $\mu(z)=0$ for $|z|>1$. Put $\mathcal{E} \equiv 1 / 2 \pi$. Then $F, G$ have to satisfy $F(z) G(z) \equiv z$ on $\partial \mathbb{D}$. Let $P_{+}$be the Riesz projection, that is, the orthogonal projection of $L^{2}(\partial \mathbb{D})$ onto $H^{2}$. Then, obviously, $P_{\mu} \mathcal{K}=\mathcal{K} P_{+}$. Therefore M4) holds in this case if and only if $P_{+}$extends to a bounded operator on $|F| L^{2}(d \nu)$, that is, if and only if $|F|$ satisfies the Muckenhoupt condition $\left(A_{2}\right)$.

So M4) has the sense of a vector Muckenhoupt condition. It implies that $P_{\mu}$ is a bounded projection on $\mathcal{K} F \mathcal{E} L^{2}(e)$. In a recent work [20] by Treil and Volberg, a very explicit form of this condition on $\partial \mathbb{D}$ has been found.

To construct more general mosaic tuples, one has to start from a piecewise analytic projection-valued function $\mu$ and then find $F, \mathcal{E}, G$, $\nu$ (see Section 5 below). 
We introduce the following Smirnov type (closed) subspaces of $\mathcal{K} F \mathcal{E} L^{2}(e)$, which will be called mosaic model spaces

$$
\begin{gathered}
E^{2}(\mu)=P_{\mu} \mathcal{K} F \mathcal{E} L^{2}(e)=\left\{u \in \mathcal{K} F \mathcal{E} L^{2}(e): u=\mu u \text { on } \mathbb{C} \backslash \gamma\right\} \\
E_{0}^{2}(1-\mu)=\left(I-P_{\mu}\right) \mathcal{K} F \mathcal{E} L^{2}(e) \\
\quad=\left\{u \in \mathcal{K} F \mathcal{E} L^{2}(e): u=(1-\mu) u \text { on } \mathbb{C} \backslash \gamma\right\}
\end{gathered}
$$

(we use the notation $|\cdot|, 1$ for the norm and the identity operator on a finite-dimensional space).

Functions in $E^{2}(\mu)$ and $E_{0}^{2}(1-\mu)$ are analytic on $\mathbb{C} \backslash \gamma$ and take value 0 at infinity; moreover, the functions in $E^{2}(\mu)$ are identically zero in the unbounded component of $\hat{\mathbb{C}} \backslash \gamma$. In general, spaces $E^{2}(\mu)$, $E_{0}^{2}(1-\mu)$ depend on the whole mosaic tuple rather than only on $\mu$.

Associated to (1.1) is the factorization

$$
p^{*}(u)=G^{*}(u) \mathcal{E}^{*}(u) F^{*}(u) .
$$

Put

$$
\overline{\mathcal{K}} g(z)=\int \frac{g(t)}{\bar{t}-\bar{z}} d \nu(t), \quad g \in G^{*} \mathcal{E} L^{2}(e)
$$

and $\overline{\mathcal{K}} G^{*} \mathcal{E} L^{2}(e)=\left\{\overline{\mathcal{K}} G^{*} \mathcal{E} g: g \in L^{2}(e)\right\}$. Then

$$
\overline{\mathcal{K}} G^{*} \mathcal{E} L^{2}(e)=\left(\mathcal{K} F \mathcal{E} L^{2}(e)\right)^{*}
$$

if we use the pairing

$$
\left\langle\mathcal{K} F \mathcal{E} f, \overline{\mathcal{K}} G^{*} \mathcal{E} g\right\rangle_{d} \stackrel{\text { def }}{=}\langle f, g\rangle, \quad f, g \in L^{2}(e) .
$$

The following fact will be proved later.

Proposition 1.1. The projection $P_{\mu}^{*}: \overline{\mathcal{K}} G^{*} \mathcal{E} L^{2}(e) \longrightarrow \overline{\mathcal{K}} G^{*} \mathcal{E} L^{2}(e)$ is given by

$$
\left(P_{\mu}^{*} v\right)(z)=\left(1-\mu^{*}(z)\right) v(z), \quad z \in \mathbb{C} \backslash \gamma
$$

The subspaces

$$
\begin{aligned}
\bar{E}_{0}^{2}\left(1-\mu^{*}\right) & =P_{\mu}^{*} \overline{\mathcal{K}} G^{*} \mathcal{E} L^{2}(e) \\
& =\left\{u \in \overline{\mathcal{K}} G^{*} \mathcal{E} L^{2}(e): u=\left(1-\mu^{*}\right) u \text { on } \mathbb{C} \backslash \gamma\right\}, \\
\bar{E}^{2}\left(\mu^{*}\right) & =\left(I-P_{\mu}^{*}\right) \overline{\mathcal{K}} G^{*} \mathcal{E} L^{2}(e) \\
& =\left\{u \in \overline{\mathcal{K}} G^{*} \mathcal{E} L^{2}(e): u=\mu^{*} u \text { on } \mathbb{C} \backslash \gamma\right\},
\end{aligned}
$$


are completely analogous to the model spaces $E^{2}(\mu), E_{0}^{2}(1-\mu)$. Functions in $\bar{E}^{2}\left(\mu^{*}\right), \bar{E}_{0}^{2}\left(1-\mu^{*}\right)$ are antianalytic on $\mathbb{C} \backslash \gamma$. Since the maps $f \longmapsto \mathcal{K} F \mathcal{E} f, f \longmapsto \overline{\mathcal{K}} G^{*} \mathcal{E} f, f \in L^{2}(e)$ are one-to-one, we will regard the four mosaic model spaces as embedded into $L^{2}(e)$.

The most simple case is the above example, where one puts $F(z) \equiv$ $z, G(z) \equiv 1$. Then $E^{2}(\mu)=H^{2}, E_{0}^{2}(1-\mu)=H_{0}^{2}(\hat{\mathbb{C}} \backslash \operatorname{clos} \mathbb{D}) \stackrel{\text { def }}{=}$ $\left\{z^{-1} f\left(z^{-1}\right): f \in H^{2}\right\}, \bar{E}^{2}\left(\mu^{*}\right)=\bar{H}^{2}, \bar{E}_{0}^{2}\left(1-\mu^{*}\right)=\bar{H}_{0}^{2}(\hat{\mathbb{C}} \backslash \operatorname{clos} \mathbb{D})$.

Definition. Subspaces $\mathcal{K}^{-1} E^{2}(\mu), \mathcal{K}^{-1} E_{0}^{2}(1-\mu)$ of $F \mathcal{E} L^{2}(e)$ and $\overline{\mathcal{K}}^{-1} \bar{E}^{2}\left(\mu^{*}\right), \overline{\mathcal{K}}^{-1} \bar{E}_{0}^{2}\left(1-\mu^{*}\right)$ of $G^{*} \mathcal{E} L^{2}(e)$ will be called the spaces of boundary values of functions in corresponding model classes $E^{2}(\mu)$, $E_{0}^{2}(1-\mu), \bar{E}^{2}\left(\mu^{*}\right), \bar{E}_{0}^{2}\left(1-\mu^{*}\right)$.

Put

$$
L(z)=(1-\mu(z)) M, \quad L^{\prime}(z)=\mu(z) M,
$$

then

$$
L(z)^{\perp}=\mu^{*}(z) M, \quad L^{\prime}(z)^{\perp}=\left(1-\mu^{*}(z)\right) M,
$$

and for each $z \in \mathbb{C} \backslash \gamma$, we have direct sum decompositions

$$
M=L(z) \dot{+} L^{\prime}(z)=L(z)^{\perp} \dot{+} L^{\prime}(z)^{\perp} .
$$

Xia uses the notation $M(z), M^{\prime}(z)$ instead of $L(z), L^{\prime}(z)$.

The functions

$$
\varphi_{t, m}(z)=\frac{\mu(z)-\mu(t)}{z-t} m, \quad \varphi_{*, t, n}(z)=\frac{\mu^{*}(z)-\mu^{*}(t)}{\bar{z}-\bar{t}} n,
$$

$t \in \mathbb{C} \backslash \gamma, m, n \in M$, will be called the Cauchy reproducing kernels.

Basic facts we need about the model spaces are collected in the following theorem.

Theorem 1.2. Let $(M, F, \mathcal{E}, G, \nu, \mu)$ be a mosaic tuple. Then

a) Direct sum decompositions

$$
\begin{aligned}
\mathcal{K} F \mathcal{E} L^{2}(e) & =E_{0}^{2}(1-\mu) \dot{+} E^{2}(\mu), \\
\overline{\mathcal{K}} G^{*} \mathcal{E} L^{2}(e) & =\bar{E}^{2}\left(\mu^{*}\right) \dot{+} \bar{E}_{0}^{2}\left(1-\mu^{*}\right),
\end{aligned}
$$


hold, $P_{\mu}$ is the parallel projection onto $E^{2}(\mu)$ with respect to the decomposition (1.8) and $P_{\mu}^{*}$ is the parallel projection onto $\bar{E}_{0}^{2}\left(1-\mu^{*}\right)$ with respect to the decomposition (1.9).

b) The following equalities with respect to duality (1.4) hold for annihilator spaces

$$
E^{2}(\mu)^{\perp}=\bar{E}^{2}\left(\mu^{*}\right), \quad E_{0}^{2}(1-\mu)^{\perp}=\bar{E}_{0}^{2}\left(1-\mu^{*}\right) .
$$
duals

c) The duality (1.4) gives rise to the following representations of

$$
\left(E^{2}(\mu)\right)^{*}=\bar{E}_{0}^{2}\left(1-\mu^{*}\right), \quad\left(E_{0}^{2}(1-\mu)\right)^{*}=\bar{E}^{2}\left(\mu^{*}\right) .
$$

d) The Cauchy kernels $\varphi_{t, m}, t \in \mathbb{C} \backslash \gamma, m \in M$ are in $\mathcal{K} F \mathcal{E} L^{2}(e)$ and generate it. The Cauchy kernels $\varphi_{*, t, n}$ generate $\overline{\mathcal{K}} G^{*} \mathcal{E} L^{2}(e)$. The reproducing formulas

$$
\left\langle u, \varphi_{*, t, n}\right\rangle_{d}=\langle u(t), n\rangle, \quad\left\langle\varphi_{t, m}, v\right\rangle_{d}=\langle m, v(t)\rangle,
$$

hold for all $t \in \mathbb{C} \backslash \gamma, m, n \in M, u \in \mathcal{K} F \mathcal{E} L^{2}(e), v \in \overline{\mathcal{K}} G^{*} \mathcal{E} L^{2}(e)$.

e) Moreover,

$$
\begin{aligned}
& \overline{\operatorname{span}}\left\{\varphi_{t, m}: t \in \mathbb{C} \backslash \gamma, m \in L^{\prime}(t)\right\}=E_{0}^{2}(1-\mu), \\
& \overline{\operatorname{span}}\left\{\varphi_{t, m}: t \in \mathbb{C} \backslash \gamma, m \in L(t)\right\}=E^{2}(\mu), \\
& \overline{\operatorname{span}}\left\{\varphi_{*, t, m}: t \in \mathbb{C} \backslash \gamma, m \in L(t)^{\perp}\right\}=\bar{E}_{0}^{2}\left(1-\mu^{*}\right), \\
& \overline{\operatorname{span}}\left\{\varphi_{*, t, m}: t \in \mathbb{C} \backslash \gamma, m \in L^{\prime}(t)^{\perp}\right\}=\bar{E}^{2}\left(\mu^{*}\right) .
\end{aligned}
$$

f) The operators $M_{z} u(z)=z u(z)$ on $E^{2}(\mu)$ and $M_{\bar{z}} u(z)=\bar{z} u(z)$ on $\bar{E}^{2}\left(\mu^{*}\right)$ are subnormal. Their adjoints are given by

$$
M_{z}^{*} v(z)=\bar{z} v(z)-\left(1-\mu^{*}(z)\right)\left(\left.\bar{z} v(z)\right|_{z=\infty}\right),
$$

where $v \in \bar{E}_{0}^{2}\left(1-\mu^{*}\right)$,

$$
M_{\bar{z}}^{*} v(z)=z v(z)-(1-\mu(z))\left(\left.z v(z)\right|_{z=\infty}\right),
$$

where $v \in E_{0}^{2}(1-\mu)$. 
Proofs of Proposition 1.1 And Theorem 1.2. It follows from (1.2) and (1.7) that

$$
\begin{aligned}
\varphi_{t, m}(z) & =\int \frac{p(s) m}{(s-z)(s-t)} d \nu(s) \\
& =\mathcal{K}\left(F(\cdot) \mathcal{E}(\cdot)(\cdot-t)^{-1} G(\cdot) m\right)(z) .
\end{aligned}
$$

Since $G(\cdot) m \in L^{2}(e)$ by M2), it follows that $\varphi_{t, m} \in \mathcal{K} F \mathcal{E} L^{2}(e)$. Similarly, $\varphi_{*, t, m} \in \overline{\mathcal{K}} G^{*} \mathcal{E} L^{2}(e)$. Take any $v \in \mathcal{K}=G^{*} \mathcal{E} L^{2}(e)$, then $v=\overline{\mathcal{K}} G^{*} \mathcal{E} g$ for some $g \in L^{2}(e)$, and by (1.14) we have

$$
\begin{aligned}
\left\langle\varphi_{t, m}, v\right\rangle_{d} & =\left\langle(\cdot-t)^{-1} G m, g\right\rangle_{L^{2}(e)} \\
& =\int\left\langle\mathcal{E}(s)(s-t)^{-1} G(s) m, g\right\rangle d \nu(s) \\
& =\langle m, v(t)\rangle,
\end{aligned}
$$

which gives the second identity in (1.10). Now suppose that $v$ is orthogonal to all reproducing kernels $\varphi_{t, m}$. Then $v(z) \equiv 0$ on $\mathbb{C} \backslash \gamma$, so that $G^{*} \mathcal{E} g \equiv 0$, which implies $g \equiv 0$ by M2). This shows the completeness of the $\varphi_{t, m}$. The first equality in (1.10) and the completeness of the $\varphi_{*, t, m}$ are proved in the same way. Thus, d) holds true.

Next, one gets from (1.7) that

$$
P_{\mu} \varphi_{t, m}=\varphi_{t,(1-\mu(t)) m} .
$$

For every $v \in \overline{\mathcal{K}} G^{*} \mathcal{E} L^{2}(e)$ and $m \in M, t \in \mathbb{C} \backslash \gamma$,

$$
\begin{aligned}
\left\langle m,\left(P_{\mu}^{*} v\right)(t)\right\rangle & =\left\langle\varphi_{t, m}, P_{\mu}^{*} v\right\rangle \\
& =\left\langle\varphi_{t,(1-\mu(t)) m}, v\right\rangle \\
& =\left\langle m,\left(1-\mu^{*}(t)\right) v\right\rangle
\end{aligned}
$$

which proves Proposition 1.1.

Now assertion a) of Theorem 1.2 is a direct consequence of the definitions of the model spaces. Next, it is obvious that

$$
\varphi_{t, m}(z)=\frac{\mu(z)-\mu(t)}{z-t} m=-\frac{(1-\mu(z))-(1-\mu(t))}{z-t} m
$$

is in $E^{2}(\mu)$ if $m \in L(t)$ and in $E_{0}^{2}(1-\mu)$ if $m \in L^{\prime}(t)$ (see (1.5)). Since $\left\{\varphi_{t, m}\right\}$ generate $\mathcal{K} F \mathcal{E} L^{2}(e),\left\{P_{\mu} \varphi_{t, m}\right\}$ generate $E^{2}(\mu)$ and so on; in this way we obtain (1.11). 
Next, $v \in E^{2}(\mu)^{\perp}$ if and only if $\langle v(t), m\rangle=\left\langle v, \varphi_{t, m}\right\rangle=0, t \in \mathbb{C} \backslash \gamma$ ,$m \in L(t)$, if and only if $v(t) \in L(t)^{\perp}=\mu(t)^{*} M, t \in \mathbb{C} \backslash \gamma$, if and only if $v \in \bar{E}^{2}\left(\mu^{*}\right)$. Similarly, $E_{0}^{2}(1-\mu)=\bar{E}_{0}^{2}\left(1-\mu^{*}\right)^{\perp}$, so that b) has been checked. The first equality in $c$ ) is a direct consequence of b) and the identity

$$
E^{2}(\mu)^{*}=\left(\mathcal{K} F \mathcal{E} L^{2}(e)\right)^{*} / E^{2}(\mu)^{\perp} .
$$

We obtain that $E_{0}^{2}(1-\mu)^{*}=\bar{E}^{2}\left(\mu^{*}\right)$ in the same way.

To prove $\mathrm{f})$, we observe that

$$
M_{z}=\left.\mathcal{M}_{z}\right|_{E^{2}(\mu)}=P_{\mu} \mathcal{M}_{z} P_{\mu}
$$

where

$$
\mathcal{M}_{z} \mathcal{K} f \stackrel{\text { def }}{=} \mathcal{K}(z f), \quad f \in F \mathcal{E} L^{2}(e) .
$$

One sees that $\mathcal{M}_{z}^{*}=\mathcal{M}_{\bar{z}}$, where

$$
\mathcal{M}_{\bar{z}} \overline{\mathcal{K}} g \stackrel{\text { def }}{=} \overline{\mathcal{K}}(\bar{z} g), \quad g \in G^{*} \mathcal{E} L^{2}(e) .
$$

Since $\mathcal{M}_{z}$ and $\mathcal{M}_{\bar{z}}$ are normal, $M_{z}$ and $M_{\bar{z}}$ are subnormal. Let $v=$ $\overline{\mathcal{K}} g \in \bar{E}_{0}^{2}\left(1-\mu^{*}\right)$. Then

$$
\begin{aligned}
M_{z}^{*} v(z) & =P_{\mu}^{*} \mathcal{M}_{\bar{z}} P_{\mu}^{*} v(z) \\
& =\left(P_{\mu}^{*} \overline{\mathcal{K}}(\bar{z} g)\right)(z) \\
& =P_{\mu}^{*}\left(\bar{z} v-\left.\left(1-\mu^{*}(z)\right)(\bar{z} v)\right|_{z=3 D \infty}\right),
\end{aligned}
$$

which proves (1.12). One proves (1.13) in the same way.

\section{A scattering type scheme.}

Suppose that $K$ is a Hilbert space and $N: K \longrightarrow K$ is a linear operator. We will apply our scheme only to situations when $\sigma(N) \stackrel{\text { def }}{=} \gamma$ has empty interior. Suppose that

Sc1) $K=H^{\prime} \dot{+} H$, where $N H \subset H$.

Sc2) There exists a finite-dimensional subspace $M$ of $H$ such that

$$
N H^{\prime} \subset H^{\prime}+M .
$$

We put $S=N \mid H$. 
In this section, we discuss tuples $\left(N, K, H^{\prime}, H, M\right)$ subject to Sc1), Sc2). Two more requirements will be added in Section 3. Our aim is to establish a relationship between tuples $\left(N, K, H^{\prime}, H, M\right)$ and mosaic tuples from Section 1.

The original Xia's model corresponds to the case when $S \in \mathcal{L}(H)$ is pure subnormal, $N \in \mathcal{L}(K)$ is its minimal normal extension, $H^{\prime}=H^{\perp}$ and $M=\left[S^{*}, S\right] H$ (see Section 6.2).

If $R$ is a linear space and $\mathcal{W}$ an open subset in $\widehat{\mathbb{C}}$, then we denote by $\operatorname{Hol}(\mathcal{W}, R)$ the space of all holomorphic functions $f: \mathcal{W} \longrightarrow R$ and by $\overline{\operatorname{Hol}}(\mathcal{W}, R)$ the corresponding space of antiholomorphic functions. If $\infty \in \mathcal{W}$, then we put $\operatorname{Hol}_{0}(\mathcal{W}, R)=\left\{f \in \operatorname{Hol}_{0}(\mathcal{W}, R): f(\infty)=0\right\}$ and define $\overline{\operatorname{Hol}}_{0}(\mathcal{W}, R)$ similarly.

Any linear operator $B: K \longrightarrow R$ gives rise to an operator $W_{B}$ : $K \longrightarrow \operatorname{Hol}_{0}(\mathbb{C} \backslash \gamma, R)$, defined by

$$
\left(W_{B} x\right)(z)=B(N-z)^{-1} x, \quad x \in K, z \in \mathbb{C} \backslash \gamma .
$$

The operator $W_{B}$ "almost diagonalizes" $N$ in the sense that

$$
\left(W_{B} N x\right)(z)=z\left(W_{B} x\right)(z)-\left(\left.z\left(W_{B} x\right)(z)\right|_{z=\infty}\right) .
$$

In what follows, we will see how to obtain "almost diagonalization" operators $W_{B}$ with good additional properties with respect to the decomposition $K=H^{\prime} \dot{+} H$.

We put

$$
\begin{gathered}
L(z)=\left\{m \in M:(N-z)^{-1} m \in H\right\} \\
L^{\prime}(z)=\left\{m \in M:(N-z)^{-1} m \in H^{\prime}\right\} \quad(z \in \mathbb{C} \backslash \gamma) .
\end{gathered}
$$

Lemma 2.1. For each $z \in \mathbb{C} \backslash \gamma$,

$$
M=L(z) \dot{+} L^{\prime}(z) \text {. }
$$

Proof. By the definition, $L(z) \cap L^{\prime}(z)=0$. Take any $m \in M$, and let $(N-z)^{-1} m=g_{1}+g_{2}$, where $g_{1} \in H, g_{2} \in H^{\prime}$. Put $l_{j}=(N-z) g_{j}$, then $l_{1}+l_{2}=m$, and $l_{1} \in H, l_{2} \in H^{\prime}+M$. It follows that $l_{2}=m-l_{1} \in$ $\left(H^{\prime}+M\right) \cap H=M, l_{1} \in M$, and we are done. 


\subsection{Transform $\widetilde{U}$.}

Let $P_{H}: K \longrightarrow H, P_{H^{\prime}}: K \longrightarrow H^{\prime}$ be the coordinate projections with respect to the decomposition given in Sc1), then $P_{H}+P_{H^{\prime}}=I$. Put

$$
A=P_{H} N P_{H^{\prime}}: K \longrightarrow M
$$

and define $\rho(z): K \longrightarrow M$ by

$$
\rho(z)=A(N-z)^{-1}, \quad z \in \mathbb{C} \backslash \gamma
$$

We define a transform $\widetilde{U}: K \longrightarrow \operatorname{Hol}(\mathbb{C} \backslash \gamma, M)$ by

$$
(\widetilde{U} x)(z)=\rho(z) x, \quad x \in K, z \in \mathbb{C} \backslash \gamma
$$

(note that $\left.\widetilde{U}=W_{N, A}\right)$.

Lemma 2.2. For all $z \in \mathbb{C} \backslash \gamma, \rho(z)^{2}=\rho(z) P_{H}$.

Proof. This is a straightforward calculation. Using that

$$
P_{H^{\prime}}(N-z) P_{H^{\prime}}=P_{H^{\prime}}(N-z),
$$

we get

$$
\begin{aligned}
\rho(z)^{2}= & P_{H} N P_{H^{\prime}}(N-z)^{-1}\left(I-P_{H^{\prime}}\right)(N-z) P_{H^{\prime}}(N-z)^{-1} \\
= & P_{H} N P_{H^{\prime}}(N-z)^{-1} \\
& -P_{H} N P_{H^{\prime}}(N-z)^{-1} P_{H^{\prime}}(N-z) P_{H^{\prime}}(N-z)^{-1} \\
= & P_{H} N P_{H^{\prime}}(N-z)^{-1}-P_{H} N P_{H^{\prime}}(N-z)^{-1} P_{H^{\prime}} \\
= & \rho(z) P_{H} .
\end{aligned}
$$

Now we define the mosaic $\mu$, associated to the tuple $\left(N, K, H^{\prime}\right.$, $H, M)$, by

$$
\mu(z)=\rho(z) \mid M: M \longrightarrow M, \quad z \in \mathbb{C} \backslash \gamma
$$


It follows from the above lemma that $\mu(z)^{2}=\mu(z)$, that is, $\mu(z)$ is a projection. It also follows that

$$
\begin{aligned}
& \left(\widetilde{U} P_{H} x\right)(z)=\mu(z)(\widetilde{U} x)(z), \\
& \left(\widetilde{U} P_{H^{\prime}} x\right)(z)=(1-\mu(z))(\widetilde{U} x)(z), \quad x \in K .
\end{aligned}
$$

Set

$$
\operatorname{Hol}(\{L\})=\{u \in \operatorname{Hol}(\mathbb{C} \backslash \gamma, M): u(z) \in L(z), z \in \mathbb{C} \backslash \gamma\},
$$

and define similarly spaces $\operatorname{Hol}\left(\left\{L^{\prime}\right\}\right), \overline{\operatorname{Hol}}\left(\left\{L^{\perp}\right\}\right)$, etc.

\section{Lemma 2.3.}

1) If $x \in H$, then $\widetilde{U} x \in \operatorname{Hol}\left(\left\{L^{\prime}\right\}\right)$.

2) If $x \in H^{\prime}$, then $\widetilde{U} x \in \operatorname{Hol}(\{L\})$.

3) The operator $\widetilde{U}$ "almost diagonalizes" $N$, that is, it satisfies

4) It diagonalizes $S=N \mid H$

$$
(\widetilde{U} S x)(z)=z(\widetilde{U} x)(z), \quad x \in H
$$

Proof. Assertions 1), 2) follow from (2.7). Equality $\widetilde{U}=W_{N, A}$ implies 3 ). Since $\mu \equiv 0$ in the unbounded connected component of $\mathbb{C} \backslash \gamma$, it follows that

$$
(\widetilde{U} N x)(z)=z \widetilde{U} x(z)-\left.(z \widetilde{U} x(z))\right|_{z=\infty}=z \widetilde{U} x(z), \quad x \in H
$$

Lemma 2.4. One has

$$
\operatorname{Ker} \mu(z)=L(z), \quad \text { Range } \mu(z)=L^{\prime}(z) .
$$

Proof. Obviously, $\mu(z) m=0$ for $m \in L(z)$. If $m \in L^{\prime}(z)$, then

$$
\mu(z) m=P_{H} N(N-z)^{-1} m=P_{H}(N-z)(N-z)^{-1} m=m .
$$


It follows from Lemma 2.1 that $\mu(z)$ is the parallel projection onto $L^{\prime}(z)$ that corresponds to the decomposition (2.5).

So the transform $\widetilde{U}$ "almost diagonalizes" $N$ and has good properties with respect to the decomposition $K=H^{\prime} \dot{+} H$ (see (2.8)). Now we shall construct a good "almost diagonalization" operator for $N^{*}$.

\subsection{Transform $\widetilde{V}$.}

The decomposition $K=H^{\prime} \dot{+} H$ gives rise to a dual decomposition

$$
K=H_{*}^{\prime} \dot{+} H_{*}
$$

where we have put $H_{*}^{\prime} \stackrel{\text { def }}{=} H^{\perp}, H_{*} \stackrel{\text { def }}{=} H^{\prime \perp}$. We will consider $H_{*}^{\prime}, H_{*}$ as realizations of the duals to $H^{\prime}, H$, respectively, and assume $S^{*}$ to be defined on $H_{*}$. Then $P_{H^{\prime}}^{*}, P_{H}^{*}$ are parallel projections onto $H_{*}^{\prime}, H_{*}$ with respect to the decomposition $(2.9)$. Since $A^{*}=P_{H^{\prime}}^{*} N^{*} P_{H}^{*}$, we have

$$
M_{*} \stackrel{\text { def }}{=} \text { Range } A^{*} \subset H_{*}^{\prime} \text {. }
$$

It is easy to see that

$$
N^{*} H_{*}^{\prime} \subset H_{*}^{\prime}, \quad N^{*} H_{*} \subset H_{*}+M_{*} .
$$

Therefore there is a certain symmetry: the tuple $\left(N, K, H^{\prime}, H, M\right)$ can be replaced by $\left(N^{*}, K, H_{*}^{\prime}, H_{*}, M_{*}\right)$, which has the same properties Sc1), Sc2). We break (a little) this symmetry and associate with $\left(N^{*}, K, H_{*}^{\prime}, H_{*}, M_{*}\right)$ an operator

$$
(\widetilde{V} y)(z)=P_{M}\left(N^{*}-\bar{z}\right)^{-1} y, \quad y \in K, z \in \mathbb{C} \backslash \gamma,
$$

here $P_{M}$ is the orthogonal projection onto $M$.

\section{Lemma 2.5.}

i) $y \in H_{*}^{\prime}$ implies $\mu^{*} V y=V y$.

ii) $y \in H_{*}$ implies $\left(1-\mu^{*}\right) V y=V y$.

iii) The following intertwining formula holds

$$
\left(\widetilde{V} N^{*} y(z)\right)(z)=\bar{z}(\widetilde{V} y(z))(z), \quad y \in H_{*}^{\prime}, z \in \mathbb{C} \backslash \gamma
$$


Proof. If $y \in H_{*}$ and $m \in M$, then $(N-z)^{-1} \mu(z) m \in H^{\prime}$ by Lemma 2.4 , which gives $\left\langle m, \mu(z)^{*}(\widetilde{V} y)(z)\right\rangle=\left\langle(N-z)^{-1} \mu(z) m, y\right\rangle=0$, so that ii) holds. Similarly, one checks that $\left(1-\mu(z)^{*}\right) \tilde{V} y(z) \equiv 0$ if $y \in H_{*}^{\prime}$, which gives i). Assertion iii) follows from i) in the same way as in Lemma 2.3.

The following formulas are immediate

$$
\begin{aligned}
& \widetilde{U}(N-\lambda)^{-1} m=\varphi_{\lambda, m}, \\
& \widetilde{V}\left(N^{*}-\bar{\lambda}\right)^{-1} P_{H^{\prime}}^{*} A^{*} m=\varphi_{*, \lambda, m}, \quad \lambda \in \mathbb{C} \backslash \gamma, m \in M .
\end{aligned}
$$

\section{A model theorem.}

In addition to Sc1), Sc2), let us assume the following conditions.

Sc3) Spaces $(N-z)^{-1} M, z \in \mathbb{C} \backslash \gamma$, as well as spaces $\left(N^{*}-\bar{z}\right)^{-1} M_{*}$, $z \in \mathbb{C} \backslash \gamma$, are complete in $K$.

Sc4) $N$ is similar to a normal operator: there exists a scalar measure $d \nu$, a $\mathcal{L}(M)$-valued Borel function $\mathcal{E}(\cdot)=\mathcal{E}(\cdot)^{*}$ and a linear isomorphism $W: K \longrightarrow L^{2}(e)$, where $d e=\mathcal{E} d \nu$, that transforms $N$ into $M_{z}$

$$
W N=M_{z} W
$$

A condition similar to Sc3) appears in [26] as a hypothesis, which is necessary for constructing dual analytic models.

Observe that $W^{*-1}: K \longrightarrow L^{2}(e)$ is an isomorphism that satisfies

$$
W^{*-1} N^{*}=M_{\bar{z}} W^{*-1} \text {. }
$$

Definition. We say that $\left(N, K, H^{\prime}, H, M\right)$ is a scattering tuple if $\left.\mathrm{Sc} 1\right)-$ Sc4) hold.

Choose (any) $\mathcal{L}(M)$-valued matrices $F(\cdot), G(\cdot)$ such that

$$
(W m)(\cdot)=G(\cdot) m,\left(W^{*-1} A^{*} m\right)(\cdot)=F^{*}(\cdot) m, \quad m \in M .
$$

Next theorem gives a relationship between scattering tuples, mosaic tuples and corresponding mosaic model spaces. 
Theorem 3.1. Let $\left(N, K, H^{\prime}, H, M\right)$ be a scattering tuple. Let $W$ : $K \longrightarrow L^{2}(e)$ be the operator from $\left.\mathrm{Sc} 4\right)$, and let $F(\cdot), G(\cdot)$ satisfy (3.3). Then the mosaic $\mu$, given by (2.6), (2.4) coincides with the function (1.2), and $(M, F, \mathcal{E}, G, \nu, \mu)$ is a mosaic tuple. The operators (2.5), (2.11) admit representations

$$
\widetilde{U}=\mathcal{K} F \mathcal{E} W, \quad \widetilde{V}=\overline{\mathcal{K}} G^{*} \mathcal{E} W^{*-1} .
$$

Moreover,

$$
\begin{gathered}
P_{\mu}=\widetilde{U} P_{H} \widetilde{U}^{-1}, \\
\langle\widetilde{U} x, \widetilde{V} y\rangle_{d}=\langle x, y\rangle, \quad x, y \in K, \\
\widetilde{U} H^{\prime}=E_{0}^{2}(1-\mu), \quad \widetilde{U} H=E^{2}(\mu), \\
\widetilde{V} H^{\prime}=\bar{E}^{2}\left(\mu^{*}\right), \quad \widetilde{V} H=\bar{E}_{0}^{2}\left(1-\mu^{*}\right) .
\end{gathered}
$$

In particular, $S=N \mid H$ is similar to the model operator

$$
\widetilde{U} S \widetilde{U}^{-1}=\left(M_{z} \text { on } E^{2}(\mu)\right) .
$$

The meaning of this theorem is reflected in the following commutative diagram.

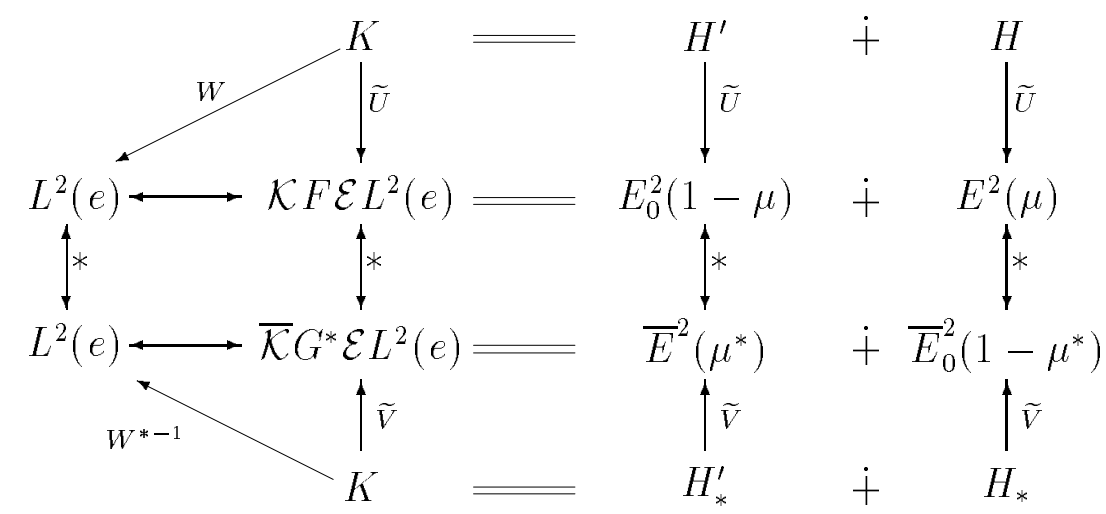

Here $\stackrel{*}{\longleftrightarrow}$ links spaces that are dual to each other. 
Using the construction of Section 5, one can give many examples of subnormal operators $S$ with $\operatorname{dim}\left[S^{*}, S\right] H=\infty$, for which this theorem produces a model with finite-dimensional "base" space $M$.

Proof of Theorem 3.1. By (3.1)-(3.3),

$$
\begin{aligned}
& W(N-\lambda)^{-1} m=(\cdot-\lambda)^{-1} G(\cdot) m, \\
& W^{*-1}\left(N^{*}-\bar{\lambda}\right)^{-1} A^{*} m=(--\bar{\lambda})^{-1} F^{*}(\cdot) m, \quad m \in M .
\end{aligned}
$$

Hence for any $m, n \in M$,

$$
\begin{aligned}
\langle\mu(\lambda) m, n\rangle & =\left\langle(N-\lambda)^{-1} m, A^{*} n\right\rangle \\
& =\left\langle W(N-\lambda)^{-1} m, W^{*-1} A^{*} n\right\rangle \\
& =\int\left\langle\mathcal{E}(z) G(z)(z-\lambda)^{-1} m, F^{*}(z) n\right\rangle d \nu(z) \\
& =\left\langle\left(\int p(z)(z-\lambda)^{-1} d \nu(z)\right) m, n\right\rangle .
\end{aligned}
$$

This implies that (1.2) holds and gives M1).

Let us show that $F(\cdot) \mid$ Range $\mathcal{E}(\cdot)$ is one-to-one $\nu$-almost everywhere. Suppose it is not so. Then there exists $f \in L^{2}(e), f \neq 0$ such that $F \mathcal{E} f=0$. By (3.10), this implies

$$
\left\langle\mathcal{E} f, W^{*-1}\left(N^{*}-\bar{\lambda}\right)^{-1} A^{*} m\right\rangle_{L^{2}(e)}=0,
$$

for all $\lambda \in \mathbb{C} \backslash \gamma, m \in M$. Then Sc3) gives that $f \perp$ Range $W^{*-1}$, a contradiction.

The second part of M2) is proved in the same way.

Condition M3) follows from Sc4) and (3.10). Also, (3.10) and (2.12) show that

$$
(\widetilde{U} x)(z)=(\mathcal{K} F \mathcal{E} W x)(z), \quad z \in \mathbb{C} \backslash \gamma,
$$

whenever $x=(N-\lambda)^{-1} m, m \in M, \lambda \in \mathbb{C} \backslash \gamma$. Therefore (3.11) holds for all $x \in K$. We obtain the second equality in (3.4) in the same way. In particular,

$$
\widetilde{U}: K \longrightarrow \mathcal{K} F \mathcal{E} L^{2}(e), \quad \widetilde{V}: K \longrightarrow \overline{\mathcal{K}} G^{*} \mathcal{E} L^{2}(e)
$$

are isomorphisms. 
Formula (3.5) follows from (2.7). Hence M4) holds. Formula (3.6) is immediate from (3.4) and (1.4). It follows that $\widetilde{V}=\widetilde{U}^{*-1}$, and therefore $P_{\mu}^{*}=\widetilde{V} P_{H}^{*} \widetilde{V}^{-1}$. Now (3.7), (3.8) follow from the results of Section 1.

Remark. Any mosaic tuple $(M, F, \mathcal{E}, G, \nu, \mu)$ can appear as a result of an application of the above theorem. If suffices to put $K=L^{2}(e)$, $H=E^{2}(\mu), H^{\prime}=E_{0}^{2}(1-\mu)$ and to embed $M$ into $E^{2}(\mu)$ according to the rule $m \longmapsto \mu(\cdot) m, m \in M$. Then $\widetilde{U}$ and $\widetilde{V}$ are identity maps.

\section{Connection with dual bundle shift models.}

Let us recall briefly the construction of [26]. Let $S: H \longrightarrow H$ be a linear operator, and choose an auxiliary operator $J: H \longrightarrow R$, where $\operatorname{dim} R<\infty$. Assume that

A1) $\operatorname{Ker}(S-z)=0$ for all $z$ in $\mathbb{C} \backslash \sigma_{\text {ess }}(S)$.

A2) $J \mid \operatorname{Ker}\left(S^{*}-\bar{z}\right)$ is one-to-one for all $z$ in $\mathbb{C} \backslash \sigma_{\text {ess }}(S)$.

Then the ultraspectrum $\mathcal{F}$ of $S$ is defined as the antianalytic family of spaces $\mathcal{F}=\left\{H(z): z \notin \sigma_{\text {ess }}(S)\right\}$, where $H(z)=J \operatorname{Ker}\left(S^{*}-\bar{z}\right)$.

In the setting of [26], the ultraspectrum is an analytic family because we used there bilinear products. See [27] for a reformulation for sesquilinear products.

Next, let $H_{*}$ be a realization of the dual space to $H$. Diagonalizing transforms

$$
U: H \longrightarrow \operatorname{Hol}\left(\left\{H(z)^{*}\right\}\right), \quad V: H_{*} \longrightarrow \overline{\operatorname{Hol}}_{0}\left(\left\{\left(H(z)^{\perp}\right)^{*}\right\}\right)
$$

were defined via the formulas

$$
\begin{gathered}
\langle(U x)(z), m\rangle=\left\langle x, h_{z, m}\right\rangle, \quad\langle(V x)(z), l\rangle=\left\langle x, g_{z, l}\right\rangle, \\
x \in H, z \in \mathbb{C} \backslash \sigma_{\mathrm{ess}}(S), m \in H(z), l \in H(z)^{\perp},
\end{gathered}
$$

where $\left\{h_{z, m}\right\},\left\{g_{z, l}\right\}$ are families of vectors in $H_{*}, H$, respectively, that are uniquely determined by the conditions

$$
\begin{aligned}
& h_{z, m} \in \operatorname{Ker}\left(S^{*}-\bar{z}\right), \quad J h_{z, m}=m \in H(z), \\
& (z-S) g_{z, l}=J^{*} l, \quad l \in H(z)^{\perp} .
\end{aligned}
$$


The vectors $g_{z, l}$ were called almost-eigenvectors of $S$. If the families $\left\{h_{z, m}\right\}$ and $\left\{g_{z, l}\right\}$ are complete, then $U$ and $V$ are one-to-one. It has been shown in [26] that $U$ transforms $S$ into the operator of multiplication by the independent variable on the model space $U H$ and $V$ transforms $S^{*}$ into the operator $v \longmapsto \bar{z} v-(\bar{z} v)(\infty)$ on the model space $V H_{*}$. There is a natural duality between $U H$ and $V H_{*}$, which is defined in an intrinsic way.

Now let us set up the relationship between the dual analytic models and the construction of sections $1-3$. Assume that $\left(N, K, H^{\prime}, H, M\right)$ is a scattering tuple, and let $S=N \mid H$. Put

$$
H_{*}=H^{\perp}, \quad R=M, \quad J=P_{M} .
$$

Proposition 4.1. For this choice of $R, J, \mathrm{~A} 1)$, A2) are satisfied. The ultraspectrum of $S$ is given by

$$
H(z)=L(z)^{\perp}=\mu^{*}(z) M .
$$

It follows that the direct sum decompositions (1.6) give rise to isomorphisms

$$
\begin{aligned}
& H(z)^{*}=\left(L(z)^{\perp}\right)^{*}=M / L(z) \cong L^{\prime}(z), \\
& \left(H(z)^{\perp}\right)^{*}=L(z)^{*}=M / L(z)^{\perp} \cong L^{\prime}(z)^{\perp} .
\end{aligned}
$$

We denote them as $i_{z}:\left(L(z)^{\perp}\right)^{*} \longrightarrow L^{\prime}(z), i_{*, z}: L(z)^{*} \longrightarrow L^{\prime}(z)^{\perp}$. Then instead of $U, V$ we can consider the transforms

$$
\widetilde{U}: H \longrightarrow \operatorname{Hol}\left(\left\{L^{\prime}(z)\right\}\right), \quad \widetilde{V}: H_{*} \longrightarrow \overline{\operatorname{Hol}}_{0}\left(\left\{L^{\prime}(z)^{\perp}\right\}\right),
$$

acting by

$$
\widetilde{U} x(z)=i_{z}(U x)(z), \quad \tilde{V} y(z)=i_{*, z}(V y)(z) .
$$

Proposition 4.2. Operators (4.3) coincide with the transforms $\widetilde{U}, \widetilde{V}$ from Section 2.

To prove Propositions 4.1 and 4.2, we need the following fact. 
Lemma 4.3. Let $z \in \mathbb{C} \backslash \sigma_{\mathrm{ess}}(S)$, and put

$$
h_{z, m}=\rho(z)^{*} m, \quad m \in L(z)^{\perp} .
$$

Then $\operatorname{Ker}\left(S^{*}-\bar{z}\right)=\left\{h_{z, m}: m \in L(z)^{\perp}\right\}$ and $J h_{z, m}=m$, so that $\left\{h_{z, m}\right\}$ is exactly the family defined in (4.1).

Proof. For any $h \in \operatorname{Ker}\left(S^{*}-\bar{z}\right),\left(N^{*}-\bar{z}\right) h \in H_{*}^{\prime}$, which gives

$$
\left(N^{*}-\bar{z}\right) h=P_{H_{*}^{\prime}} N^{*} h=A^{*} h=A^{*} P_{M} h .
$$

Therefore $h=\rho(z)^{*} P_{M} h=\rho(z)^{*} \mu(z)^{*} P_{M} h=h_{z, m}$, where $m \stackrel{\text { def }}{=}$ $\mu(z)^{*} P_{M} h \in L(z)^{\perp}$. Next, by $(2.10)$,

$$
\left(S^{*}-\bar{z}\right) h_{z, m}=P_{H_{*}}\left(N^{*}-\bar{z}\right) h_{z, m}=P_{H_{*}} A^{*} m=0,
$$

for all $m \in L(z)^{\perp}$. At last, $J h_{z, m}=P_{M} \rho(z)^{*} m=\mu(z)^{*} m=m$ for $m \in L(z)^{\perp}$.

Proof of Propositions 4.1 and 4.2. Since $S=N \mid H$, A1) holds. Lemma 4.3 implies A2) and (4.2). Therefore

$$
\begin{gathered}
\langle U x(z), m\rangle=\langle\rho(z) x, m\rangle=\langle\widetilde{U} x(z), m\rangle, \\
\langle V y(z), l\rangle=\left\langle y,(z-N)^{-1} l\right\rangle=\langle\widetilde{V} y(z), l\rangle,
\end{gathered}
$$

for $m \in H(z)=L(z)^{\perp}, l \in H(z)^{\perp}=L(z)$. This proves Proposition 4.2 .

We also obtain the following fact.

Proposition 4.4. Eigenvectors $h_{z, m}$ of $S^{*}\left(z \in \mathbb{C} \backslash \sigma_{\mathrm{ess}}(S)\right)$ are complete. Almost-eigenvectors $g_{z, l}$ of $S\left(z \in \mathbb{C} \backslash \sigma_{\mathrm{ess}}(S)\right)$ are also complete.

Proof. $\widetilde{U}: H \longrightarrow E^{2}(\mu)$ and $\widetilde{V}: H_{*} \longrightarrow \bar{E}_{0}^{2}\left(1-\mu^{*}\right)$ are isomorphisms. Therefore $U$ and $V$ are one-to-one. 


\section{How to construct mosaic tuples?}

The method we give here is not the most general, but it shows how much freedom we have.

Let $\gamma$ be a finite union of arbitrary $C^{1}$-smooth arcs, intersecting only in their endpoints, such that $\mathbb{C} \backslash \gamma$ has at least two connected components. Denote the components of $\mathbb{C} \backslash \gamma$ by $\Omega_{1}, \ldots, \Omega_{t}$. We assume for simplicity that for any of the arcs $\alpha$ that compose $\gamma$, one has $\alpha \subset$ $\partial \Omega_{j} \cap \partial \Omega_{k}$ for some $j \neq k$. Let $\mu: \mathbb{C} \backslash \gamma \longrightarrow \mathcal{L}(M)$ be a projectionvalued analytic function such that $\mu \equiv 0$ in the unbounded component and for each $\Omega_{k}, \mu$ extends to a continuous function on clos $\Omega_{k}$. Fix an orientation of each of the arcs of $\gamma$. For a function $f$ in $\operatorname{Hol}(\mathbb{C} \backslash \gamma)$, we denote by $f_{i}, f_{e}$ its interior and exterior limit values on $\gamma$.

Put $d \nu=\left.|d z|\right|_{\gamma}$ and $p_{1}=\mu_{i}-\mu_{e}$. Then (1.2) holds for

$$
p=\left.\frac{1}{2 \pi i} p_{1} \frac{d z}{|d z|}\right|_{\gamma} .
$$

Assume additionally that $\operatorname{rank} p$ is constant on each of the $\operatorname{arcs} \partial \Omega_{j} \cap$ $\partial \Omega_{k}$ and that

$$
\left\|p(z) \mid(\operatorname{Ker} p(z))^{\perp}\right\|>\varepsilon>0
$$

for $z$ in the interior of these arcs, where $\varepsilon$ does not depend on $z$. To assure that $p$ satisfies this property one can take for $\mu$, for instance, a small perturbation of a locally constant mosaic $\mu_{0}$ "in general position".

Next let us fix a factorization (1.1) of $p$. By (5.1), we may assume that $F, F^{-1}, G, G^{-1}$ are in $L^{\infty}(\gamma ; M)$ and that $\mathcal{E}$ is projection-valued. We will use the Smirnov classes $E^{2}\left(\Omega_{j}\right)$; we refer to [6], [17] for their definition. The corresponding classes $E^{2}\left(\Omega_{j} ; M\right)$ of $M$-valued functions are defined componentwise.

It is easy to see that

$$
\begin{aligned}
\mathcal{K} F \mathcal{E} L^{2}(e)=\left\{u \in \oplus_{j} E^{2}\left(\Omega_{j} ; M\right):\right. \\
u(\infty)=0 \text { and } u_{i}(\cdot)-u_{e}(\cdot) \in p(\cdot) M \\
\text { almost everywhere on } \gamma\} .
\end{aligned}
$$

Let $u \in \mathcal{K} F \mathcal{E} L^{2}(e)$, then $u_{i}-u_{e}=p_{1} f$ for some $f \in L^{2}(d \nu ; M)$. Therefore $\mu u \in \oplus_{j} E^{2}\left(\Omega_{j} ; M\right)$, and we have

$$
\mu_{i} u_{i}-\mu_{e} u_{e}=p_{1}\left(u_{i}+\left(1-\mu_{i}\right) f\right) .
$$


Hence M4) holds. It follows that all conditions M1)-M4) hold.

In this example, $E^{2}(\mu)$ does not depend on the choice of factorization (1.1) of $p$, and

$$
\begin{aligned}
& E^{2}(\mu)=\left\{\left\{i_{z} u(z)\right\}: u \in \operatorname{Mod}^{2}(\mathcal{F})\right\}, \\
& \bar{E}_{0}^{2}\left(1-\mu^{*}\right)=\left\{\left\{i_{*, z} v(z)\right\}: v \in \overline{\operatorname{Mod}}_{0}^{2}\left(\mathcal{F}_{\perp}\right)\right\},
\end{aligned}
$$

where $\operatorname{Mod}^{2}(\mathcal{F}), \overline{\operatorname{Mod}}_{0}^{2}\left(\mathcal{F}_{\perp}\right)$ are, essentially, the Smirnov type model spaces from [26, Section 2] (one has to define $\overline{\operatorname{Mod}}_{0}^{2}\left(\mathcal{F}_{\perp}\right)$ as a space of antianalytic functions, see [27]).

We can make the following résumé. If only the family $\{H(z)\}=$ $\left\{\mu(z)^{*} M\right\}$ is given, then only the dual model spaces $U H, V H_{*}$ appear that correspond to the operators $S$ on $H$ and $S^{*}$ on $H_{*}$. If the whole mosaic $\mu$ is given, then all four model spaces $E_{0}^{2}(1-\mu), E^{2}(\mu), \bar{E}^{2}\left(\mu^{*}\right)$, $\bar{E}_{0}^{2}\left(1-\mu^{*}\right)$ appear. They serve to model spaces $H^{\prime}, H, H_{*}^{\prime}, H_{*}$.

\section{Xia's model.}

Here we show how to specialize the constructions of sections 2 and 3 in order to obtain Xia's results.

\subsection{The discriminant surface.}

For the reader's convenience, we remind the definitions and results from [25] we will need. Some of the notions discussed are reflected on the Figure.

Let $S: H \longrightarrow H$ be a subnormal operator of finite type and $N: K \longrightarrow K$ its minimal normal extension. Define $M, C, \Lambda$ by $(0.1)$ [11]. Let $\Delta$ be the discriminant curve (0.2), $\widehat{\Delta}$ its blow-up [11] and $\widehat{\Delta}=\widehat{\Delta}_{1} \cup \cdots \cup \widehat{\Delta}_{T}$ the decomposition of $\widehat{\Delta}$ into irreducible components. In order to reflect the multiplicities of $\widehat{\Delta}_{j}$, sometimes we will write $\widehat{\Delta}=\widehat{\Delta}_{1}^{k_{1}} \cup \cdots \cup \widehat{\Delta}_{T}^{k_{T}}$ [25]. Each of $\widehat{\Delta}_{j}$ is a compact Riemann surface, and we consider them as branched coverings of the $z$-plane.

Let $\Delta_{0}$ be the set of regular points of $\Delta$. Then $\Delta \backslash \Delta_{0}$ is finite, and $\widehat{\Delta}$ is obtained from $\Delta_{0}$ by adding a finite number of points. There is a natural projection of $\widehat{\Delta}$ onto $\Delta$, which is identical on $\Delta_{0}$. The point in $\widehat{\mathbb{C}}^{2}$ that corresponds to a point $\delta \in \widehat{\Delta}$ will be denoted as $(z(\delta), w(\delta))$, and we write $\delta \sim(z(\delta), w(\delta))$. 
Let

$$
\sigma_{C}(\Lambda)=\left\{z \in \mathbb{C}: \operatorname{det}\left(C-\left(w-\Lambda^{*}\right)(z-\Lambda)\right)=0, \text { for all } w \in \mathbb{C}\right\} .
$$

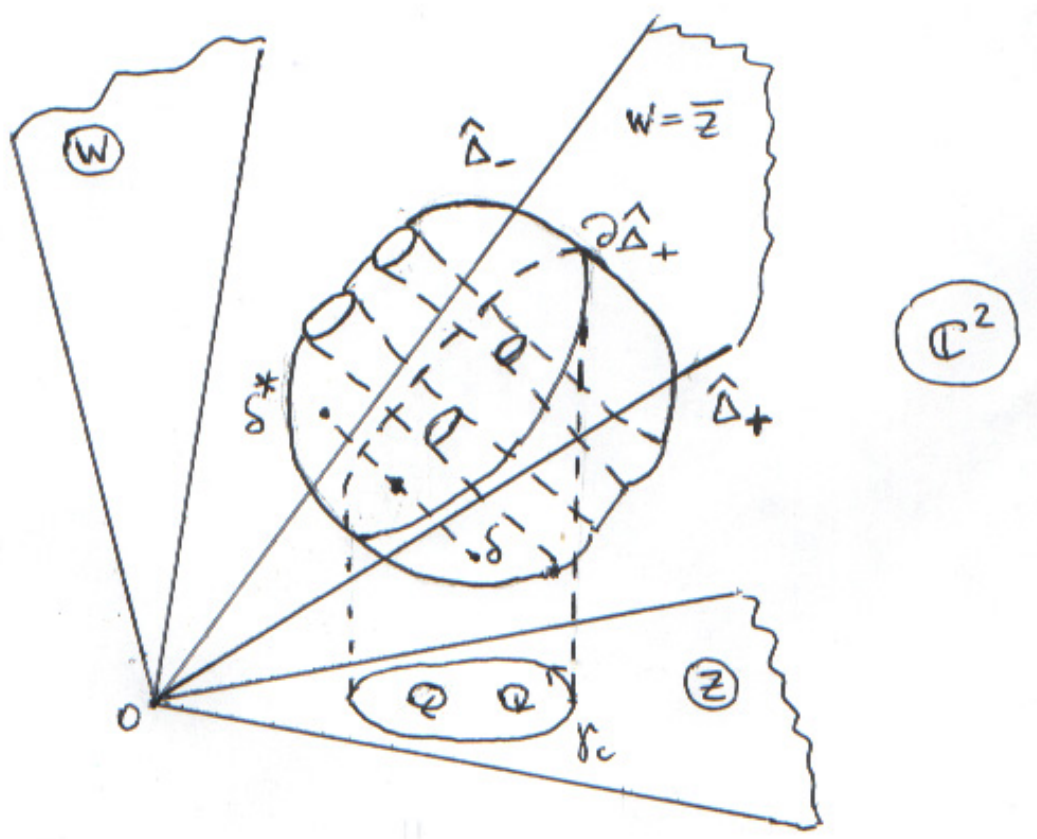

Figure.

It is immediate that $\sigma_{C}(\Lambda) \subset \sigma(\Lambda)$. For any $z_{0} \in \sigma_{C}(\Lambda), \widehat{\Delta}$ has irreducible components $z \equiv z_{0}$ and $w \equiv \bar{z}_{0}$. These irreducible components of $\widehat{\Delta}$ were called degenerate and all other irreducible components were called nondegenerate. Let $\widehat{\Delta}_{\text {deg }}$ be the union of degenerate components of $\widehat{\Delta}$ and $\widehat{\Delta}_{\text {ndeg }}$ the union of nondegenerate components.

Let

$$
\widehat{\Delta}_{+}=\left\{\delta \in \widehat{\Delta}_{\text {ndeg }}:|\eta|<1\right\}, \quad \widehat{\Delta}_{-}=\left\{\delta \in \widehat{\Delta}_{\text {ndeg }}:|\eta|>1\right\},
$$

where $\eta$ is a meromorphic function on $\widehat{\Delta}_{\text {ndeg }}$, defined by $\eta=-d z / d w$. The map $\delta=(z, w) \longmapsto \delta^{*}=(\bar{w}, \bar{z})$ is an antianalytic involution on $\Delta_{0}$, and it naturally extends to $\widehat{\Delta}$. It interchanges $\widehat{\Delta}_{+}$and $\widehat{\Delta}_{-}$, because $\eta\left(\delta^{*}\right)=\overline{\eta(\delta)}^{-1}, \delta \in \widehat{\Delta}_{\text {ndeg }}$.

The curve $\widehat{\Delta}$ was called separated if the set $\widehat{\Delta} \cap\{(z, \bar{z}): z \in \mathbb{C}\}$ divides each nondegenerate component $\widehat{\Delta}_{k}$ into two connected components; then these two connected components are $\widehat{\Delta}_{k} \cap \widehat{\Delta}_{+}$and $\widehat{\Delta}_{k} \cap$ 
$\widehat{\Delta}_{-}$. In this case, $\partial \widehat{\Delta}_{+}=\left\{\delta \in \widehat{\Delta}: \delta=\delta^{*}\right\}$ (we refer to [25] for more details).

Let

$$
\widehat{\Delta}^{\prime}=\widehat{\Delta}_{\mathrm{ndeg}} \cup \bigcup_{\lambda \in \sigma_{C}(\Lambda)}\{(z, w): w \equiv \bar{\lambda}\}
$$

be the algebraic curve obtained from $\widehat{\Delta}$ by excluding from it the "vertical" components $z \equiv \lambda$. A projection-valued meromorphic function $\delta \longmapsto Q(\delta)$ on $\widehat{\Delta}^{\prime}$ is defined by

$$
Q((z, w))=-\frac{1}{2 \pi i} \int_{\partial \mathcal{D}_{w}}\left(C(z-\Lambda)^{-1}+\Lambda^{*}-u\right)^{-1} d u, \quad z \notin \sigma(\Lambda),
$$

where $\mathcal{D}_{w}$ is a small disc centered in $w$ such that $\mathcal{D}_{w} \cap \sigma\left(C(z-\Lambda)^{-1}+\right.$ $\left.\Lambda^{*}\right)=\{w\}$. We have

$$
\begin{array}{cl}
Q\left(\delta_{1}\right) Q\left(\delta_{2}\right)=0, & \delta_{1} \neq \delta_{2}, z\left(\delta_{1}\right)=z\left(\delta_{2}\right), \\
\sum_{z(\delta)=\zeta} Q(\delta)=I, & \text { for all } \zeta \in \mathbb{C} \backslash \sigma(\Lambda) .
\end{array}
$$

Put

$$
\gamma_{c}=z\left(\partial \widehat{\Delta}_{+}\right) \subset \mathbb{C}, \quad \gamma=\{z \in \mathbb{C}:(z, \bar{z}) \in \Delta\} .
$$

Then $\gamma_{c}$ is a union of analytic arcs and $\gamma \backslash \gamma_{c}$ is finite. It follows from [21]-[23] that

$$
\gamma_{c} \subset \sigma(N) \subset \gamma
$$

Orient the curve $\gamma_{c}$ according to the positive orientation of the boundary $\partial \widehat{\Delta}_{+}$of $\widehat{\Delta}_{+}$. One can define a function $\xi$ on $\gamma_{c}$ (except for a finite number of points) so that $d z=i \xi(z)|d z|$ and $|\xi| \equiv 1$ almost everywhere on $\gamma_{c}$. Then $\eta((z, \bar{z})) \equiv \xi(z)^{2}$ almost everywhere on $\gamma_{c}$.

Denote by $\delta_{\zeta}$ the delta-measure at a point $\zeta$ of $\mathbb{C}$. The following theorem collects some of the results of [22], [25].

Theorem A. Let $(C, \Lambda)$ correspond to a subnormal operator $S$ of finite type. Then $\widehat{\Delta}$ is separated and all Jordan blocks of $C(z-\Lambda)^{-1}+\Lambda^{*}$ that correspond to eigenvalues $w$ such that $(z, w) \in \widehat{\Delta}_{\text {ndeg }}$ are trivial for all but a finite number of values of $z$. The involution $\delta \longmapsto \delta^{*}$ maps each of the nondegenerate components of $\widehat{\Delta}$ onto itself. The mosaic (2.6) has a representation

$$
\mu(z)=\sum_{(z, w) \in \widehat{\Delta}_{+}} Q((z, w)) .
$$


Moreover, $\operatorname{rank} Q(\delta)=k_{j}, \delta \in \widehat{\Delta}_{j} \cap \Delta_{0}$.

There is a finite subset $R$ of $\mathbb{C}$ and matrices $A_{s}, s \in R$ such that $d e(\cdot)=\mathcal{E}(\cdot) d \nu(\cdot)$, where $d \nu=\left.|d z|\right|_{\gamma_{c}}+\sum_{\zeta \in R} \delta_{\zeta}$ and

$$
\mathcal{E}(s)= \begin{cases}\frac{1}{2 \pi} \xi(s)(s-\Lambda)^{-1} Q((s, \bar{s})), & s \in \gamma_{c} \backslash R \\ A_{s}, & s \in R .\end{cases}
$$

\subsection{Xia's model as a particular case of mosaic model spaces.}

Let $S, N$ be as in Section 6.1, and put $H^{\prime}=H^{\perp}, M=\left[S^{*}, S\right] H$. It is known that $S^{\prime}=N^{*} \mid H^{\prime}$ also is a subnormal operator of finite type. Let $E(\cdot)$ be the spectral measure of $N$, and define $e$ by $e(\cdot)=$ $P_{M} E(\cdot) \mid M$. In [21], Xia considers a unitary operator

$$
W: K \longrightarrow L^{2}(e)
$$

given by

$$
W f(N) m=f(\cdot) m, \quad m \in M,
$$

where $f$ is any bounded Borel function on $\sigma(N)$. In our terminology, we get the situation of sections 2 and 3 , where now $H^{\prime} \perp H$ and $W^{*-1}=W$ satisfies both (3.1), (3.2).

Lemma 6.1. $\left(K, N, H^{\prime}, H, M\right)$ is a scattering tuple.

Proof. Properties Sc1) and Sc4) are obvious. It is easy to see that $\left[S^{*}, S\right]=A^{*} A$, where $A$ is defined by (2.3). Therefore $P_{H} N H^{\prime}=$ $\left[S^{*}, S\right] H$, which gives (Sc2). It follows, for instance, from [15, Chapter 2, Theorem 1.3] that $\operatorname{span}\left\{S^{n} M: n \geqslant 0\right\}=H$. This implies the first part of Sc3). The second part of Sc3) is proved in [23, Lemma 1].

Since

$$
W A^{*} m=W\left(N^{*}-S^{*}\right) m=\left({ }^{-}-\Lambda^{*}\right) m, \quad m \in M,
$$

we see that (3.3) holds if we put

$$
F(z)=z-\Lambda, \quad G(z) \equiv I .
$$


So now the model spaces are $\mathcal{K}(\cdot-\Lambda) \mathcal{E} L^{2}(e)$ and $\overline{\mathcal{K}} \mathcal{E} L^{2}(e)$. We arrive at the following result, which, besides the representation (6.2) of $\mu$, is essentially contained in Xia's works [21] and [23].

Theorem 6.2. Let $S$ be any subnormal operator of finite type. Define $F, G$ by (6.5), $\mu, \mathcal{E}, \nu$ from Theorem $\mathrm{A}$ and $W$ by (6.4). Then $W$ is unitary, and all conclusions of Theorem 3.1 hold. In particular, $\mu$ is alternatively given by

$$
\mu(z)=\int \frac{(t-\Lambda) d e(t)}{t-z} .
$$

Now we have $H_{*}^{\prime}=H^{\prime}, H_{*}=H$, so that diagram (3.9) acquires the form

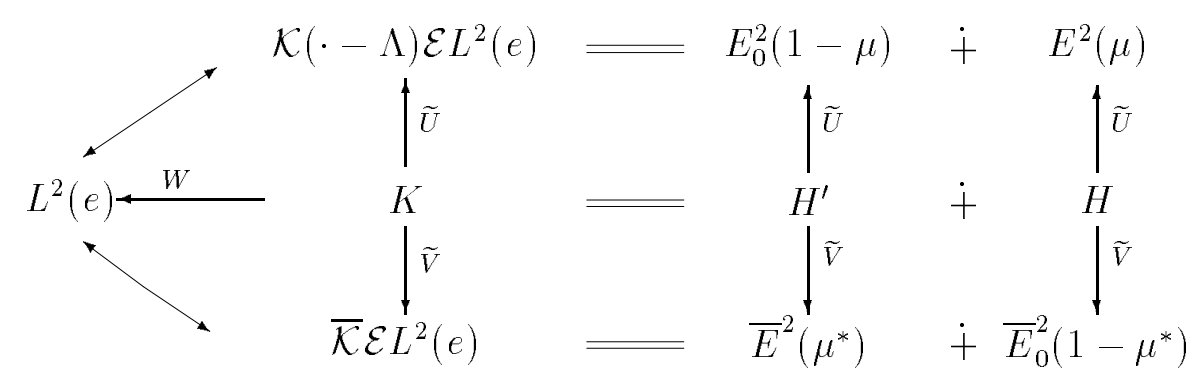

Xia calls the function $\widetilde{U} x(z)$ the analytic representation of a vector $x \in K$ and the function $\widetilde{V} x(\bar{z})$ the dual analytic representation of $x$.

Theorem 6.2 gives an explicit construction of a finite type subnormal operator from matrices $C, \Lambda$. The set of possible pairs $(C, \Lambda)$ has been completely described in [25]. If a pair $(C, \Lambda)$ satisfies the criterium that was given there, define $\mathcal{E}$, de and $\mu$ from Theorem $\mathrm{A}$ and $F, G$ from (6.5). Then $S$ will be unitarily equivalent to the operator $M_{z}$ on $E^{2}(\mu)$.

Looking at diagram (6.7), one notices an interesting phenomenon. The operator $j: \mathcal{K}(\cdot-\Lambda) \mathcal{E} L^{2}(e) \longrightarrow \overline{\mathcal{K}} \mathcal{E} L^{2}(e)$, given by

$$
j \mathcal{K}(\cdot-\Lambda) h \stackrel{\text { def }}{=} \overline{\mathcal{K}} h, \quad h \in \mathcal{E} L^{2}(e),
$$

is obviously an isometric isomorphism. One sees from diagram (6.7) that

$$
j=\widetilde{V} \widetilde{U}^{-1}
$$


Therefore the multiplication operator

$$
h \longmapsto(\cdot-\Lambda) h
$$

maps isomorphically $\overline{\mathcal{K}}^{-1} \bar{E}^{2}\left(\mu^{*}\right)$ onto $\mathcal{K}^{-1} E_{0}^{2}(1-\mu)$ and $\overline{\mathcal{K}}^{-1} \bar{E}_{0}^{2}\left(1-\mu^{*}\right)$ onto $\mathcal{K}^{-1} E^{2}(\mu)$. So the multiplication by a linear analytic matrix binomial maps certain spaces of boundary values of antianalytic functions onto spaces of boundary values of analytic functions.

The subsequent exposition is organized as follows. In Section 7, we replace the model operator $M_{z}$ on $E^{2}(\mu)$ (see Theorem 3.1) with the operator of multiplication by $z(\cdot)$ on a function space $H_{\mu}^{2}\left(X_{+}\right)$, which consists of analytic cross-sections of a bundle $X_{+}$over $\widehat{\Delta}_{+}$. In Section 8 , we relate the above-described phenomenon with the involution on $\Delta$. In Section 9, we will give a necessary background on weighted Hardy classes over Riemann surfaces. These facts will permit us to give a complete characterization of $H_{\mu}^{2}\left(X_{+}\right)$and to prove main structure results.

\section{External Riemann surface models.}

$$
\begin{aligned}
& \text { Put } \\
& X(\delta)=\text { Range } Q(\delta), \quad Y(\delta)=\operatorname{Range} Q^{*}(\delta), \quad \delta \in \widehat{\Delta}^{\prime} .
\end{aligned}
$$

For any component $\widehat{\Delta}_{j}$ of $\widehat{\Delta}^{\prime}, X \mid \widehat{\Delta}_{j}\left(Y \mid \widehat{\Delta}_{j}\right)$ can be considered as an analytic (antianalytic) vector subbundle of dimension $k_{j}$ of the trivial bundle $\widehat{\Delta}_{j} \times M$, where $k_{j}$ is the multiplicity of $\widehat{\Delta}_{j}$. This can be deduced from the following simple fact.

Proposition 7.1. Let $\Omega$ be a domain in $\mathbb{C}, \lambda_{0} \in \Omega, k \in \mathbb{N}$, and $r_{1}, \ldots, r_{k}: \Omega \rightarrow M$ be analytic functions such that $r_{1}(\lambda), \ldots, r_{k}(\lambda)$ are linearly independent for some $\lambda \in \Omega$. Then there exist analytic functions $q_{1}, \ldots, q_{k}$, defined in some disk $\mathcal{D}$, with $\lambda_{0} \in \mathcal{D} \subset \Omega$, such that $\overline{\operatorname{span}}\left\{r_{1}(\lambda), \ldots, r_{k}(\lambda)\right\}=\overline{\operatorname{span}}\left\{q_{1}(\lambda), \ldots, q_{k}(\lambda)\right\}$ for $\lambda \in \mathcal{D} \backslash\left\{\lambda_{0}\right\}$ and $q_{1}(\lambda), \ldots, q_{k}(\lambda)$ are linearly independent for all $\lambda \in \mathcal{D}$.

Proof. The family $\left\{r_{j}\right\}$ can be transformed into the family $\left\{q_{j}\right\}$ by taking linear combinations with constant coefficients and dividing several times by $\lambda-\lambda_{0}$. We omit the details. 
We put $\widehat{\Delta}_{-}^{\prime}=\widehat{\Delta}_{-} \cup \bigcup_{\lambda \in \sigma_{C}(\Lambda)}\{\delta: w(\delta)=\bar{\lambda}\}$, then $\widehat{\Delta}^{\prime}$ decomposes into a disjoint union

$$
\widehat{\Delta}^{\prime}=\widehat{\Delta}_{+} \cup \widehat{\Delta}_{-}^{\prime} \cup \partial \widehat{\Delta}_{+}
$$

Let

$$
X_{+}=X\left|\widehat{\Delta}_{+}, \quad Y_{+}=Y\right| \widehat{\Delta}_{+}, \quad X_{-}=X\left|\widehat{\Delta}_{-}^{\prime}, \quad Y_{-}=Y\right| \widehat{\Delta}_{-}^{\prime} .
$$

Set

$$
\begin{gathered}
a u(\delta)=Q(\delta) u(z(\delta)), \quad u \in \mathcal{K}(\cdot-\Lambda) \mathcal{E} L^{2}(e), \\
b v(\delta)=Q^{*}(\delta) v(z(\delta)), \quad v \in \overline{\mathcal{K}} \mathcal{E} L^{2}(e)
\end{gathered}
$$

$\left(\delta \in \widehat{\Delta}^{\prime}\right)$, and

$$
\begin{aligned}
H_{\mu}^{2}\left(X_{+}\right) & =a E^{2}(\mu), & H_{\mu}^{2}\left(X_{-}\right) & =a E_{0}^{2}(1-\mu), \\
\bar{H}_{\mu^{*}}^{2}\left(Y_{+}\right) & =b \bar{E}^{2}\left(\mu^{*}\right), & \bar{H}_{\mu^{*}}^{2}\left(Y_{-}\right) & =b \bar{E}_{0}^{2}\left(1-\mu^{*}\right) .
\end{aligned}
$$

It follows from (6.2) that functions in $H_{\mu}^{2}\left(X_{+}\right), \bar{H}_{\mu^{*}}^{2}\left(Y_{+}\right)$vanish on $\widehat{\Delta}_{-}^{\prime}$ and functions in $H_{\mu}^{2}\left(X_{-}\right), \bar{H}_{\mu^{*}}^{2}\left(Y_{-}\right)$vanish on $\widehat{\Delta}_{+}$. We have

$$
\begin{aligned}
& a \mathcal{K}(\cdot-\Lambda) \mathcal{E} L^{2}(e)=H_{\mu}^{2}\left(X_{-}\right)+H_{\mu}^{2}\left(X_{+}\right) \\
& b \overline{\mathcal{K}} \mathcal{E} L^{2}(e)=\bar{H}_{\mu^{*}}^{2}\left(Y_{-}\right)+\bar{H}_{\mu^{*}}^{2}\left(Y_{+}\right)
\end{aligned}
$$

We will need notation for several exceptional sets. Let $\mathrm{Pol}_{Q}$ be the set of all poles of $Q$ on $\widehat{\Delta}^{\prime}$ and $\tilde{\tau}$ the maximum of orders of these poles. Let

$$
B=z\left(\operatorname{Pol}_{Q}\right) \cup z\left(\widehat{\Delta}^{\prime} \backslash \Delta_{0}\right) \cup R
$$

where $R$ is the set from (6.3), and put $B^{\#}=z^{-1}(B) \cap \widehat{\Delta}^{\prime}$. The sets $\mathrm{Pol}_{Q}, B$ and $B^{\#}$ are finite.

Lemma 7.2. Each function in a $\mathcal{K}(\cdot-\Lambda) \mathcal{E} L^{2}(e)\left(b \overline{\mathcal{K}} \mathcal{E} L^{2}(e)\right)$ is analytic (antianalytic) on $\widehat{\Delta} \backslash\left(\partial \widehat{\Delta}_{+} \cup \mathrm{Pol}_{Q}\right)$ and has poles in points of $\mathrm{Pol}_{Q} \backslash \partial \widehat{\Delta}_{+}$ at most of order $\tilde{\tau}$. 
A priori, functions in $a \mathcal{K}(\cdot-\Lambda) \mathcal{E} L^{2}(e)$ and $b \overline{\mathcal{K}} \mathcal{E} L^{2}(e)$ may have jumps on the whole preimage curve $z^{-1}\left(\gamma_{c}\right)$. This lemma shows that they have jumps only on $\partial \widehat{\Delta}_{+}$. Hence the model spaces $H_{\mu}^{2}\left(X_{+}\right)$, $H_{\mu}^{2}\left(X_{-}\right)$consist of meromorphic cross-sections of $X_{+}, X_{-}$, respectively, and $\bar{H}_{\mu^{*}}^{2}\left(Y_{+}\right), \bar{H}_{\mu^{*}}^{2}\left(Y_{-}\right)$consist of conjugate meromorphic cross-sections of $Y_{+}, Y_{-}$. Lemma 7.2 will be proved at the end of this section.

Operations $a$ and $b$ are invertible. By (6.1), the inverses are given by

$$
\left(a^{-1} f\right)(\zeta)=\sum_{z(\delta)=\zeta} f(\delta), \quad\left(b^{-1} g\right)(\zeta)=\sum_{z(\delta)=\zeta} g(\delta), \quad \zeta \in \mathbb{C} \backslash \gamma
$$

Define Hilbert norms on $a \mathcal{K}(\cdot-\Lambda) \mathcal{E} L^{2}(e)$ and $b \overline{\mathcal{K}} \mathcal{E} L^{2}(e)$ so that $a, b$ become unitary operators. We arrive at the following fact.

Proposition 7.3. $S$ is unitarily equivalent to the operator of multiplication by $z(\cdot)$ on $H_{\mu}^{2}\left(X_{+}\right)$, and $S^{\prime}$ is unitarily equivalent to the operator of multiplication by $\bar{z}(\cdot)$ on $\bar{H}_{\mu^{*}}^{2}\left(Y_{+}\right)$.

We call these representations the external Riemann surface representations of $S, S^{\prime}$. By (6.1), projections $P_{\mu}$ and $I-P_{\mu}^{*}$ in these representations are expressed as

$$
\begin{aligned}
& a P_{\mu} a^{-1}=\left(M_{\chi_{+}} \text {on } a \mathcal{K}(\cdot-\Lambda) \mathcal{E} L^{2}(e)\right), \\
& b\left(I-P_{\mu}\right) b^{-1}=\left(M_{\chi_{+}} \text {on } b \overline{\mathcal{K}} \mathcal{E} L^{2}(e)\right),
\end{aligned}
$$

where $\chi_{+}=1$ on $\widehat{\Delta}_{+}$and $\chi_{+}=0$ on $\widehat{\Delta}_{-}^{\prime}$.

Proof of Lemma 7.2. For any domain $\mathcal{W}$ in $\mathbb{C}$, bounded by a piecewise smooth Jordan curve, the Smirnov class $E^{2}(\mathcal{W})$ has the following properties [6], [17]:

1)

$$
f(\cdot)=\frac{1}{2 \pi i} \int g(z)((\cdot)-z)^{-1} d z
$$

is in $E^{2}(\mathcal{W})$ for every $g \in L^{2}(\partial \mathcal{W},|d z|)$.

2) Each $f \in E^{2}(\mathcal{W})$ has boundary values almost everywhere on $\partial \mathcal{W}$, and

$$
\frac{1}{2 \pi i} \int f(z)(z-w)^{-1} d z
$$


gives $f(w)$ for $w \in \mathcal{W}$ and 0 for $w \in \mathbb{C} \backslash \operatorname{clos} \mathcal{W}$.

3) For any smooth arc $\gamma \subset \mathcal{W}$, the map $f \longmapsto f \mid \gamma$ from $E^{2}(\mathcal{W})$ into $L^{2}(\gamma,|d z|)$ is bounded.

Let $f \in L^{2}(e)$ and $g=\mathcal{K}(\cdot-\Lambda) \mathcal{E} f, u=a g$. By (6.3) and the Privalov-Plemelj "jump" formula [17],

$$
u_{i}(\delta)-u_{e}(\delta)=Q(\delta) Q((z, \bar{z})) f(z), \quad \delta=(z, w) \in z^{-1}\left(\gamma_{c}\right)
$$

Bearing in mind (6.1), we see that $u_{i}=u_{e}$ almost everywhere on $z^{-1}\left(\gamma_{c}\right) \backslash \partial \widehat{\Delta}_{+}$. Note that $\mathcal{E}^{1 / 2} f \mid \gamma_{c} \backslash B$ is in $L^{2}\left(|d z| \mid \gamma_{c}, M\right)$ and $(\cdot-$ $\Lambda) \mathcal{E}^{1 / 2}$ is bounded on $\gamma_{c} \backslash \mathcal{U}$ for any neighbourhood $\mathcal{U}$ of $B$. It follows that any $\delta_{0} \in z^{-1}\left(\gamma_{c}\right) \backslash\left(\partial \widehat{\Delta}_{+} \cup B^{\#}\right), \delta_{0}=\left(z_{0}, w_{0}\right)$, has a small neighbourhood $\mathcal{W}$ in $\widehat{\Delta}$ that projects homeomorphically onto a disc centered in $z_{0}$ such that $u \circ\left(z \mid \mathcal{W}^{\prime}\right)^{-1} \in E^{2}\left(z\left(\mathcal{W}^{\prime}\right)\right)$ for any connected component $\mathcal{W}^{\prime}$ of $\mathcal{W} \backslash z^{-1}\left(\gamma_{c}\right)$. The above fact 2$)$ easily implies that $u$ is a restriction of a function, analytic in a neighbourhood of $\delta_{0}$. So $u$ is analytic on $\Delta^{\prime} \backslash\left(\partial \widehat{\Delta}_{+} \cup B^{\#}\right)$.

Now let $\delta_{0} \in B^{\#} \backslash \partial \widehat{\Delta}_{+}, \delta_{0} \sim\left(z_{0}, w_{0}\right)$. Take a small neighbourhood $\mathcal{W}$ of $\delta_{0}$ with analytic boundary such that $\operatorname{clos} \mathcal{W} \cap B=\left\{\delta_{0}\right\}$. The above proof and 3) show that the map $u \longmapsto u \mid \partial \mathcal{W}$ is bounded from $a \mathcal{K}(\cdot-$ $\Lambda) \mathcal{E} L^{2}(e)$ into $L^{2}(\partial \mathcal{W},|d z|, M)$. Pick any function $s$, holomorphic on $\operatorname{clos} \mathcal{W}$, such that $s Q$ is also holomorphic on $\operatorname{clos} \mathcal{W}$. We assert that functions $s u$ are analytic at $\delta_{0}$ for all $u \in a \mathcal{K}(\cdot-\Lambda) \mathcal{E} L^{2}(e)$. Indeed, it suffices to check that this is true for $u$ in a complete set. By (1.11), the functions

$$
\varphi_{t, m}(z)=\frac{\mu(z)}{z-t} m,
$$

where $t \in \mathbb{C} \backslash \gamma$ and $m \in M$ are complete in $\mathcal{K}(\cdot-\Lambda) \mathcal{E} L^{2}(e)$. Therefore the functions $u(\delta)=Q(\delta)(z(\delta)-t)^{-1} m$ are complete in $a \mathcal{K}(\cdot-$ $\Lambda) \mathcal{E} L^{2}(e)$. For every such $u, s u$ is analytic at $\delta_{0}$.

It follows that the statement of Lemma is true for $a \mathcal{K}(\cdot-\Lambda) \mathcal{E} L^{2}(e)$. The proof for $b \overline{\mathcal{K}} \mathcal{E} L^{2}(e)$ is similar.

\section{The role of the symmetry on $\widehat{\Delta}$.}

Lemma 8.1 The identity

$$
(z-\Lambda)^{-1} Q(\delta)=\eta(\delta)^{-1} Q^{*}\left(\delta^{*}\right)\left(w-\Lambda^{*}\right)^{-1}, \quad \delta=(z, w)
$$


holds on $\widehat{\Delta}_{\text {ndeg }}$.

Proof. We use arguments similar to those of Xia [22, p. 895]. Let $\delta_{0}=\left(z_{0}, w_{0}\right) \in \widehat{\Delta}_{\text {ndeg }} \cap \Delta_{0}$ and $\mathcal{V}$ be a small neighbourhood of $\delta_{0}$ in $\mathbb{C}^{2}$. We assume that $\mathcal{V} \cap \Delta$ is given by equations $w=\alpha(z), z=\beta(w)$, where $\alpha, \beta$ are analytic functions. Then the equation of $\left\{\delta^{*}: \delta \in \mathcal{V} \cap \Delta\right\}$ is $w=\bar{\beta}(\bar{z})$. Since all Jordan blocks of $C(z-\Lambda)^{-1}+\Lambda^{*}$ that correspond to eigenvalue $\alpha(z)$ are trivial by Theorem $\mathrm{A}$, analytic perturbation theory [4] gives

$$
\left.(w-\alpha(z))\left(C-\left(w-\Lambda^{*}\right)(z-\Lambda)\right)^{-1}\right|_{(z, w) \in \mathcal{V} \cap \Delta}=-(z-\Lambda)^{-1} Q((z, \alpha(z))),
$$

the matrix on the left hand side being in fact analytic in $\mathcal{V}$. Let us apply this equality to a neighbourhood of $\delta_{0}^{*}$, pass to the adjoints and then substitute $w \longmapsto \bar{z}, z \longmapsto \bar{w}$. We obtain that

$$
\begin{aligned}
(z-\beta(w))\left(C-\left(w-\Lambda^{*}\right)(z-\Lambda)\right)^{-1} & \left.\right|_{(z, w) \in \mathcal{V} \cap \Delta} \\
& =-Q^{*}((\bar{w}, \beta(\bar{w})))\left(w-\Lambda^{*}\right)^{-1} .
\end{aligned}
$$

It remains only to remark that $\alpha\left(z_{0}\right)=w_{0}, \beta\left(w_{0}\right)=z_{0}$, and

$$
\lim _{\substack{(z, w) \in \mathbb{C}^{2} \backslash \Delta \\(z, w) \rightarrow \delta_{0}}} \frac{w-\alpha(z)}{z-\beta(w)}=-\left.\frac{d w}{d z}\right|_{\delta_{0}}=\eta\left(\delta_{0}\right)^{-1} .
$$

Let $v \in \bar{H}_{\mu^{*}}^{2}\left(Y_{-}\right)$and $u \in H_{\mu}^{2}\left(X_{-}\right)$. We define symmetries $\alpha_{1}, \alpha_{2}$ by

$$
\begin{aligned}
& \left(\alpha_{1} v\right)(\delta)=\eta(\delta)^{-1}(z(\delta)-\Lambda) v\left(\delta^{*}\right) \\
& \left(\alpha_{2} u\right)(\delta)=\bar{\eta}(\delta)^{-1}(\bar{w}(\delta)-\Lambda) u\left(\delta^{*}\right)
\end{aligned}
$$

for $\delta \in \widehat{\Delta}_{+}$(notice that then $\delta^{*} \in \widehat{\Delta}_{-} \subset \widehat{\Delta}^{\prime}$ ). By (8.1) and Lemma $7.2, \alpha_{1} v$ is a meromorphic cross-section of $X_{+}$and $\alpha_{2} u$ is a conjugate meromorphic cross-section of $Y_{+}$.

Theorem 8.2. Let $S$ be a subnormal operator of finite type. Define $F$, $G$ by (6.5). Let $\mu, \mathcal{E}, \nu, e$ be as in Theorem 6.2 and $j$ be defined by 
(6.8). Then the diagram

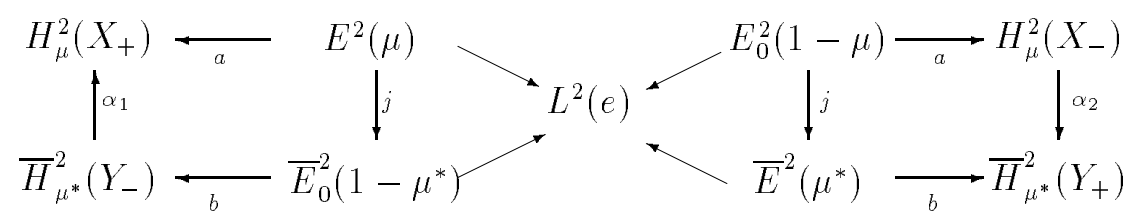

is commutative (the embeddings of the mosaic model spaces into $L^{2}(e)$ are defined by diagram (6.7)). In partucular, $j$ maps isometrically $E^{2}(\mu)$ onto $\bar{E}_{0}^{2}\left(1-\mu^{*}\right)$ and $E_{0}^{2}(1-\mu)$ onto $\bar{E}^{2}\left(\mu^{*}\right)$. Spaces $E^{2}(\mu)$ and $\bar{E}_{0}^{2}\left(1-\mu^{*}\right)$ coincide as subspaces of $L^{2}(e)$. Spaces $E_{0}^{2}(1-\mu)$ and $\bar{E}^{2}\left(\mu^{*}\right)$ also coincide as subspaces of $L^{2}(e)$.

The symmetries $\alpha_{1}, \alpha_{2}$ also explain the existence of the formulas for action of $S$ and $S^{*}$ in both model spaces $E^{2}(\mu), \bar{E}_{0}^{2}\left(1-\mu^{*}\right)$ (see [22, Theorems 2 and 3]).

Proof. Let $\delta \in \widehat{\Delta}_{+}, \delta \sim(z, w), m \in M$, and $\lambda \in \mathbb{C},(z, \bar{\lambda}) \notin \Delta$. Since $\widehat{\Delta}_{+} \subset \widehat{\Delta}_{\text {ndeg }}$, the assertion about Jordan blocks in Theorem A implies that

$$
\left(C(z-\Lambda)^{-1}+\Lambda^{*}-\bar{\lambda}\right) Q(\delta)=Q(\delta)(w-\bar{\lambda}),
$$

and together with (8.1) and the formula $\eta\left(\delta^{*}\right)=\overline{\eta(\delta)}^{-1}$ this gives

$$
\begin{aligned}
Q^{*}(\delta)\left(C-\left(\bar{z}-\Lambda^{*}\right)(\right. & \lambda-\Lambda))^{-1} m \\
= & (\bar{w}-\lambda)^{-1} Q^{*}(\delta)\left(\bar{z}-\Lambda^{*}\right)^{-1} m \\
& =(\bar{w}-\lambda)^{-1} \eta\left(\delta^{*}\right)(\bar{w}-\Lambda)^{-1} Q\left(\delta^{*}\right) m .
\end{aligned}
$$

By the results of Section 1, the elements $\varphi_{\lambda, m} \operatorname{span} \mathcal{K}(\cdot-\Lambda) \mathcal{E} L^{2}(e)$. Since $\varphi_{\lambda, m}=\mathcal{K}(\cdot-\Lambda)(\cdot-\lambda)^{-1} m$,

$$
j \varphi_{\lambda, m}(z)=\int \frac{d e(t)}{(t-\lambda)(\bar{t}-\bar{z})} m
$$

We will make use of Xia's formula

$$
\begin{aligned}
\int \frac{d e(t)}{(t-\lambda)(\bar{t}-\bar{z})}= & \mu(z)^{*}\left(C-\left(\bar{z}-\Lambda^{*}\right)(\lambda-\Lambda)\right)^{-1} \\
& -\left(C-\left(\bar{z}-\Lambda^{*}\right)(\lambda-\Lambda)\right)^{-1}(1-\mu(\lambda)),
\end{aligned}
$$


which is valid whenever $\lambda, z \notin \sigma(N)$ and $(\lambda, \bar{z}) \notin \Delta$ (see [22]). Consider two subfamilies of the family of functions $\left\{\varphi_{\lambda, m}\right\}$.

1) Let $\lambda \notin \sigma(N)$ and $m \in(1-\mu(\lambda)) M$. Then $\varphi_{\lambda, m}=(z-$ $\lambda)^{-1} \mu(z) m$ by (1.7), and by (8.5), (8.6),

$$
j \varphi_{\lambda, m}(z)=-\left(1-\mu(z)^{*}\right)\left(C-\left(\bar{z}-\Lambda^{*}\right)(\lambda-\Lambda)\right)^{-1} m .
$$

Since $j \varphi_{\lambda, m} \in \overline{\mathcal{K}} \mathcal{E} L^{2}(e)$, it follows that $j \varphi_{\lambda, m} \in \bar{E}_{0}^{2}\left(1-\mu^{*}\right)$. Moreover, by $(6.2), Q^{*}(\delta)\left(1-\mu(z(\delta))^{*}\right)=Q^{*}(\delta)$ for $\delta=(z, w) \in \widehat{\Delta}_{+}$. By (8.4),

$$
\begin{aligned}
\left(b j \varphi_{\lambda, m}\right)(\delta) & =Q^{*}(\delta)\left(C-\left(\bar{z}(\delta)-\Lambda^{*}\right)(\lambda-\Lambda)\right)^{-1} m \\
& =(\bar{w}-\lambda)^{-1} \eta\left(\delta^{*}\right)(\bar{w}-\Lambda)^{-1} Q\left(\delta^{*}\right) m
\end{aligned}
$$

so that

$$
\left(\alpha_{1} b j \varphi_{\lambda, m}\right)(\delta)=(z-\lambda)^{-1} Q(\delta) m=\left(a \varphi_{\lambda, m}\right)(\delta), \quad \delta \in \widehat{\Delta}_{+} .
$$

Since the family of functions $\varphi_{\lambda, m}$ we are considering is complete in $E^{2}(\mu)$, we conclude that $j E^{2}(\mu) \subset \bar{E}_{0}^{2}\left(1-\mu^{*}\right)$ and that the left rectangle in the diagram is commutative. Then

2) Let $\lambda \notin \sigma(N)$ and $m \in \mu(\lambda) M, \varphi_{\lambda, m}=-(z-\lambda)^{-1}(1-\mu(z)) m$.

$$
j \varphi_{\lambda, m}(z)=\mu(z)^{*}\left(C-\left(\bar{z}-\Lambda^{*}\right)(\lambda-\Lambda)\right)^{-1} m,
$$

so that $j \varphi_{\lambda, m} \in \bar{E}^{2}\left(\mu^{*}\right)$. Now we obtain from (8.4) that for $\delta \in \widehat{\Delta}_{+}$, $\delta \sim(z, w)$,

$$
\begin{aligned}
\left(b j \varphi_{\lambda, m}(\delta)\right) & =Q^{*}(\delta)\left(C-\left(\bar{z}-\Lambda^{*}\right)(\lambda-\Lambda)\right)^{-1} m \\
& =(\bar{w}-\lambda)^{-1} \eta\left(\delta^{*}\right)(\bar{w}-\Lambda)^{-1} Q\left(\delta^{*}\right) m \\
& =\alpha_{2} a \varphi_{\lambda, m}(\delta) .
\end{aligned}
$$

This proves that $j E_{0}^{2}(1-\mu) \subset \bar{E}^{2}\left(\mu^{*}\right)$ and that the right rectangle in the diagram is commutative.

At last, note that $\mathcal{K}(\cdot-\Lambda) \mathcal{E} L^{2}(e)=E^{2}(\mu) \oplus E_{0}^{2}(1-\mu)$ implies $\overline{\mathcal{K}} \mathcal{E} L^{2}(e)=j E^{2}(\mu) \oplus j E_{0}^{2}(1-\mu)$. Since $j E^{2}(\mu) \subset \bar{E}_{0}^{2}\left(1-\mu^{*}\right)$ and $j E_{0}^{2}(1-\mu) \subset \bar{E}^{2}\left(\mu^{*}\right)$, we have $j E^{2}(\mu)=\bar{E}_{0}^{2}\left(1-\mu^{*}\right)$ and $j E_{0}^{2}(1-\mu)=$ $\bar{E}^{2}\left(\mu^{*}\right)$. 
Remarks. Suppose we start from a pair $(C, \Lambda)$ that satisfies the criterium of [25, Theorem 2] and our aim is to construct the corresponding $S$. Then (6.2), (6.6) define the same function $\mu$, and model spaces $E^{2}(\mu), \bar{E}^{2}\left(\mu^{*}\right)$, etc. arise. The proof of (8.6) in [21] uses only (6.6) and the formula

$$
\left(C-\left(\bar{z}-\Lambda^{*}\right)(z-\Lambda)\right) d e(z) \equiv 0,
$$

which follows from the hypotheses on $C, \Lambda$. So the fact that $E^{2}(\mu)$ and $\bar{E}_{0}^{2}\left(1-\mu^{*}\right)$ define the same subspace of $L^{2}(e)$ can be deduced directly from the hypotheses of [25, Theorem 2]. Therefore we can put $S$ to be equal to $M_{z}$ on $E^{2}(\mu)$, and we will get all its necessary properties.

It is easier to understand the sense of Theorem 8.2 when $\widehat{\Delta}$ has no degenerate components. Then formulas (8.2), (8.3) permit us to define $\alpha_{1}^{-1}, \alpha_{2}^{-1}$ as well. But $\alpha_{1}, \alpha_{2}$ are isomorphisms even if there are degenerate components. In this case, we conclude from (8.2), (8.3) that the value of every function in $\bar{H}_{\mu^{*}}^{2}\left(Y_{-}\right)$or $H_{\mu}^{2}\left(X_{-}\right)$on degenerate components $w \equiv$ const is determined by its values on other components.

\section{Analytic functional classes over Riemann surfaces.}

This section has an auxiliary character. Our exposition uses the approaches of [1], [10].

Let $R$ be a (connected) branched Riemann surface over $\mathbb{C}$, whose boundary $\partial R$ is a finite union of analytic arcs. We assume that $\bar{R}=$ $R \cup \partial R$ is compactly imbedded into a larger Riemann surface $\widetilde{R}$ and that $\partial R=\partial \bar{R}$. Let $\delta \longmapsto z(\delta)$ be the projection of $R$ onto $\mathbb{C}$. We assume that $z(\bar{R})$ is a compact set in $\mathbb{C}$.

From now on, let us fix a base point $\delta_{0} \in R$, and let $\omega$ be the harmonic measure for $\partial R$ at $\delta_{0}$. It is easy to see that $\omega$ and the arc length measure $|d z(\cdot)|$ are mutually absolute continuous.

The Nevanlinna class of $R$ is defined as

$$
\mathcal{N}(R)=\{f \in \operatorname{Hol}(R): \log |f| \text { has a harmonic majorant }\} .
$$

The hypotheses on $R$ imply that the unit disc $\mathbb{D}$ is its universal covering space. Let

$$
\mathbf{T}: \mathbb{D} \longrightarrow R
$$

be a covering map, normalized so that $\mathbf{T}(0)=\delta_{0}$. The boundary of $R$ has a finite number of connected components, and the fundamental 
group $\pi_{1}(R)$ of $R$ is finitely generated. It follows that there exists a relatively open and dense subset $\mathcal{A}$ of $\partial \mathbb{D}$ such that $\mathbf{T}$ extends continuously to a map from $\mathcal{A}$ onto $\partial R$. (see [7]). This map will be also denoted by $\mathbf{T}$. Moreover, $\omega(E)=(2 \pi)^{-1} m_{1}\left(\mathbf{T}^{-1}(E)\right)$ for each Borel subset $E$ of $\partial R$; here $m_{1}$ is the arc length measure on $\partial \mathbb{D}$.

It is easy to see (using the techniques of [10, Section 3.4]) that $f \in \mathcal{N}(R)$ if and only if $f \circ \mathbf{T} \in \mathcal{N}(\mathbb{D})$. Since $f \in \mathcal{N}(R)$ implies $f \mid \mathcal{D} \in \mathcal{N}(\mathcal{D})$ for every connected subdomain $\mathcal{D}$ of $R$, it follows that each function $f$ in $\mathcal{N}(R)$ has non-tangential limit values $\omega$-almost everywhere on $\partial R$, and that $\mathbf{T}$ lifts these boundary values to boundary values of $f \circ \mathbf{T}$ on $\partial \mathbb{D}$. Let

$$
\mathcal{N}^{+}(\mathbb{D})=\left\{g h^{-1}: g, h \in H^{\infty}, g \text { is outer in } \mathbb{D}\right\}
$$

be the Smirnov subclass of the Nevanlinna class on $\mathbb{D}$, and define the Smirnov class of $R$ by

$$
\mathcal{N}^{+}(R)=\left\{f \in \mathcal{N}(R): f \circ \mathbf{T} \in \mathcal{N}^{+}(\mathbb{D})\right\}
$$

For each natural number $k$, we denote by $\mathcal{N}^{+}\left(R, \mathbb{C}^{k}\right)$ the set of $\mathbb{C}^{k}$ valued functions on $R$, whose components are in $\mathcal{N}^{+}(R)$.

Let $\Omega$ be a Borel measurable selfadjoint nonnegative $k \times k$ matrix function on $\partial R$, which is log-integrable, that is,

$$
\int \log ^{+} \max \left\{\|\Omega\|,\left\|\Omega^{-1}\right\|\right\} d \omega<\infty
$$

(here $\left.\log ^{+}(\cdot)=\max \{\log (\cdot), 0\}\right)$. Consider the weighted space

$$
L^{2}(\partial R, \omega, \Omega)
$$

with the norm given by $\|f\|^{2}=\int\langle\Omega f, f\rangle d \omega$ and the corresponding weighted $H^{2}$-space

$$
H^{2}(R ; \Omega)=\left\{f \in \mathcal{N}^{+}\left(R, \mathbb{C}^{k}\right): f \mid \partial R \in L^{2}(\partial R, \omega, \Omega)\right\}
$$

Let $\widetilde{\Omega}=\Omega \circ \mathbf{T}$ be the matrix weight on $\partial \mathbb{D}$ that corresponds to $\Omega$; then (9.1) gives

$$
\int \log ^{+} \max \left\{\|\widetilde{\Omega}\|,\left\|\widetilde{\Omega}^{-1}\right\|\right\}|d z|<\infty
$$


Hence there exists a matrix function $\mathcal{O}$ on $\mathbb{D}$ such that $\mathcal{O}, \mathcal{O}^{-1} \in$ $\mathcal{N}^{+}\left(\mathbb{D}, \mathbb{C}^{k \times k}\right)$ and

$$
\widetilde{\Omega}=\mathcal{O}^{*} \mathcal{O} \text { on } \partial \mathbb{D}
$$

(see [19, Chapter 5, Section 7]). The matrix function $\mathcal{O}$ is determined uniquely, up to a constant left unitary factor.

Let Möb $(\mathbb{D})$ be the group of all linear fractional transformations of $\mathbb{D}$, and let $G$ be the group of deck transformations, that is,

$$
G=\{\varphi \in \operatorname{Möb}(\mathbb{D}): \mathbf{T} \circ \varphi=\mathbf{T}\} .
$$

It is known that $G$ is a discrete group and is isomorphic to $\pi_{1}(R)$. Denote by $\mathbf{U}\left(\mathbb{C}^{k}\right)$ the group of unitary linear transformations of $\mathbb{C}^{k}$. Since $\widetilde{\Omega} \circ \varphi=\widetilde{\Omega}$ for all $\varphi \in G$, one deduces from (9.2) that for every $\varphi \in G$ there exists a constant matrix $\alpha(\varphi) \in \mathbf{U}\left(\mathbb{C}^{k}\right)$ such that

$$
\mathcal{O} \circ \varphi=\alpha(\varphi) \mathcal{O}
$$

It follows that $\alpha: G \longrightarrow \mathbf{U}\left(\mathbb{C}^{k}\right)$ is a group homomorphism.

Definition. $\alpha$ is called the character that corresponds to the matrix weight $\Omega$. $\mathbf{U}\left(\mathbb{C}^{k}\right)$.

Denote by $\operatorname{Char}_{k}(R)$ the set of all group homomorphisms $\alpha: G \longrightarrow$

Let $\alpha$ be an arbitrary element of $\operatorname{Char}_{k}(R)$. If an analytic $\mathbb{C}^{k}-$ or $\mathbb{C}^{k \times k}$ - valued function $f$ satisfies $f \circ \varphi=\alpha(\varphi) f, \varphi \in G$, then $f$ is called $\alpha$-automorphic. Put

$$
H_{\alpha}^{2}(R)=\left\{f \in H^{2}\left(\mathbb{D}, \mathbb{C}^{k}\right): f \text { is } \alpha \text {-automorphic }\right\},
$$

it is a closed subspace of $H^{2}\left(\mathbb{D}, \mathbb{C}^{k}\right)$. Informally, we interpret an element of $H_{\alpha}^{2}(R)$ as a multivalued analytic function $f$ on $R$ such that $|f|$ is single-valued.

Lemma 9.1. Let $\Omega, \widetilde{\Omega}, \mathcal{O}$ be as above, and let $\alpha$ be the character that corresponds to $\Omega$. Then the map

$$
f \longmapsto \mathcal{O} \cdot(f \circ \mathbf{T})
$$

defines an isometric isomorphism of $H^{2}(R, \Omega)$ onto $H_{\alpha}^{2}(R)$. 
Proof. Let $f \in \operatorname{Hol}\left(R, \mathbb{C}^{k}\right)$. Then $f \in \mathcal{N}^{+}\left(R, \mathbb{C}^{k}\right)$ if and only if $\mathcal{O} \cdot(f \circ \mathbf{T}) \in \mathcal{N}^{+}\left(\mathbb{D}, \mathbb{C}^{k}\right)$. Since for $f \in \mathcal{N}^{+}\left(R, \mathbb{C}^{k}\right)$,

$\int_{\partial R}\langle\Omega f, f\rangle d \omega=\int_{\partial \mathbb{D}}\langle\widetilde{\Omega}(f \circ \mathbf{T}),(f \circ \mathbf{T})\rangle \frac{|d z|}{2 \pi}=\int_{\partial \mathbb{D}}\|\mathcal{O} \cdot(f \circ \mathbf{T})\|^{2} \frac{|d z|}{2 \pi}$,

the above map is an isomorphic isomorphism of $H^{2}(R, \Omega)$ onto its image in $H^{2}\left(\mathbb{D}, \mathbb{C}^{k}\right)$. A function $g \in \mathcal{N}^{+}\left(\mathbb{D}, \mathbb{C}^{k}\right)$ is $\alpha$-automorphic if and only if $g=\mathcal{O} \cdot(f \circ \mathbf{T})$ for some $f \in \mathcal{N}^{+}\left(R, \mathbb{C}^{k}\right)$ (here we use that the least harmonic majorant of a $G$-invariant subharmonic function is also $G$-invariant). Therefore the image of our map is exactly $H_{\alpha}^{2}(R)$.

Lemma 9.2. The operator $M_{z(\cdot)}$ of multiplication by $z(\cdot)$ on

$$
L^{2}(\partial R, \omega, \Omega)
$$

is the minimal normal extension of the operator of multiplication by $z(\cdot)$ on $H^{2}(R, \Omega)$.

It suffices to prove that

$$
\underset{\lambda \notin z(\partial R)}{\overline{\operatorname{span}}}\left\{(z(\cdot)-\lambda)^{-1} f \mid \partial R: f \in H^{2}(R, \Omega)\right\}=L^{2}(\partial R, \omega, \Omega) .
$$

Let $\psi \in L^{2}(\partial R, \omega, \Omega)$ be orthogonal to all functions in the left hand part. Then, by the Hartogs-Rosenthal theorem [12],

$$
\sum_{\substack{\delta \in \partial R \\ z(\delta)=\zeta}}\langle\Omega(\delta) \psi(\delta), f(\delta)\rangle=0
$$

for almost every $\zeta \in z(\partial R)$ and all $f \in H^{2}(R, \Omega)$. Take any $g \in$ $H^{2}(R, \Omega)$ and put here $f=\varphi g, \varphi \in H^{\infty}(R)$. Since for each $\zeta \in z(\partial R)$ we can choose $\varphi$ which is analytic on a neighbourhood of $\bar{R}$ and has arbitrarily prescribed values in points of $z^{-1}(\zeta) \cap \partial R$, we conclude that $\psi=0$ almost everywhere on $\partial R$.

Define an operator $S_{R, \alpha}$ on $H_{\alpha}^{2}(R)$ by

$$
S_{R, \alpha} f=(z \circ \mathbf{T}) \cdot f
$$


It is obviously subnormal. In our interpretation of $H_{\alpha}^{2}(R)$ as a space of multivalued functions on $R, S_{R, \alpha}$ acts as multiplication by $z(\cdot)$. Sometimes we will write $S_{R, \alpha, \delta_{0}}$ to show the dependence on the base point $\delta_{0}$.

Functions in $H_{\alpha}^{2}(R)$, considered as multivalued functions on $R$, have boundary limit values on $\partial R$, which are also multivalued. Let us fix a Borel subset $E$ of $\partial \mathbb{D}$ such that $\mathbf{T} \mid E$ is an isomorphism of $E$ onto $\partial R$, and put $\mathbf{T}^{[-1]}=(\mathbf{T} \mid E)^{-1}$. We associate with each $f \in H_{\alpha}^{2}(R)$ the single-valued function $f \circ \mathbf{T}^{[-1]}$ on $\partial R$.

One has

$$
\|f\|_{H_{\alpha}^{2}(R)}^{2}=\int_{\partial R}\left|f \circ \mathbf{T}^{[-1]}\right|^{2} d \omega
$$

(we use here the norm in $H^{2}$, given by $\|x\|^{2}=(2 \pi)^{-1} \int|x|^{2}|d z|$.) So the map $f \longmapsto f \circ \mathbf{T}^{[-1]}$ allows us to consider $H_{\alpha}^{2}(R)$ as embedded isometrically into $L^{2}\left(\partial R, \omega, \mathbb{C}^{k}\right)$.

With this agreement, the map $f \longmapsto \mathcal{O} \circ \mathbf{T}^{[-1]} \cdot f$ extends the map of Lemma 9.1 to an isometric isomorphism of $L^{2}(\partial R, \omega, \Omega)$ onto $L^{2}\left(\partial R, \omega, \mathbb{C}^{k}\right)$. Therefore, by Lemma 9.2 , the operator $M_{z(\cdot)}$ of multiplication by $z(\cdot)$ on $L^{2}\left(\partial R, \omega, \mathbb{C}^{k}\right)$ is the minimal normal extension of the operator $S_{R, \alpha}$.

From now on, we assume that the Riemann surface $\widetilde{R}$, which contains $\bar{R}$, is such that the imbedding $R \subset \widetilde{R}$ induces an isomorphism between the fundamental groups $\pi_{1}(R)$ and $\pi_{1}(\widetilde{R})$.

Lemma 9.3. For any $\alpha \in \operatorname{Char}_{k}(R)$, there exists an $\alpha$-automorphic function $A: \mathbb{D} \longrightarrow \mathbb{C}^{k}$ such that $\|A\|,\left\|A^{-1}\right\|$ are bounded in $\mathbb{D}$.

The proof of Lemma 9.3 will be given a little bit later.

Let $\mathbb{I}$ be the unit element of $\operatorname{Char}_{k}(R)$, that is, $\mathbb{I}(\varphi)=I$ for all $\varphi \in$ $G$. Then $H_{\mathbb{I}}^{2}(R)$ is the set of $G$-invariant functions in $H^{2}(\mathbb{D})$; this space is naturally isometrically isomorphic to the unweighted Hardy space $H^{2}(R)=H^{2}(R, I)$. In the situation of Lemma 9.3, the map $f \longmapsto A \cdot f$, $f \in H_{\mathbb{I}}^{2}(R)$, defines a (not necessarily isometric) isomorphism of $H_{\mathbb{I}}^{2}(R)$ onto $H_{\alpha}^{2}(R)$. Since this isomorphism commutes with $M_{z(\cdot)}$, we obtain the following fact.

Corollary. All the operators $S_{R, \alpha}, \alpha \in \operatorname{Char}_{k}(R)$, are mutually similar. 
Putting $\mathcal{O}=A$ in the construction preceding Lemma 9.1, we also see that each operator $S_{R, \alpha}$ is unitarily equivalent to an operator $M_{z(\cdot)}$ on $H^{2}(R, \Omega)$ for a matrix weight $\Omega$ such that $\|\Omega\|,\left\|\Omega^{-1}\right\|$ are uniformly bounded on $R$.

Definition. Characters $\alpha, \beta$ in $\operatorname{Char}_{k}(R)$ are called equivalent $(\alpha \sim \beta)$ if there is a constant $u$ in $\mathbf{U}\left(\mathbb{C}^{k}\right)$ such that $\beta(\varphi)=u \alpha(\varphi) u^{*}$ for all $\varphi \in G$.

The following statement is in a sense analogous to [1, Theorem 6$]$.

Lemma 9.4. Suppose that there is a point in $\mathbb{C}$ whose preimage on $R$ consists of exactly one point. Let $\alpha, \beta \in \operatorname{Char}_{k}(R)$. The operators $S_{R, \alpha}$ and $S_{R, \beta}$ are unitarily equivalent if and only if $\alpha \sim \beta$.

Proof of Lemma 9.3. Let $P: \mathcal{A} \longrightarrow \widetilde{R}$ be a universal covering map for $\widetilde{R}$; we assume $\mathcal{A}$ to be a simply connected domain in $\mathbb{C}$. Put $\widetilde{\mathbb{D}}=P^{-1}(R)$, then $\widetilde{\mathbb{D}}$ is connected and simply connected and $P \mid \widetilde{\mathbb{D}}$ is the universal covering map for $R$. If $\varphi: \mathbb{D} \longrightarrow \widetilde{\mathbb{D}}$ is a conformal map such that $P(\varphi(0))=\delta_{0}$, then we can set $\mathbf{T}=P \circ \varphi$. Let $\widetilde{G}$ be the group of deck transformations of $\widetilde{R}$. It is easy to see that the map $g \longmapsto \varphi^{-1} \circ(g \mid \widetilde{\mathbb{D}}) \circ \varphi$ is an isomorphism of $\widetilde{G}$ onto $G$; it gives rise to a canonical isomorphism between $\operatorname{Char}_{k}(R)$ and $\operatorname{Char}_{k}(\widetilde{R})$.

Let $\gamma$ be the element of $\operatorname{Char}_{k}(\widetilde{R})$ that corresponds to $\alpha$. There exists a $\mathbb{C}^{k \times k}$-valued $\gamma$-automorphic function $\Gamma$ on $\mathcal{A}$ such that $\operatorname{det} \Gamma \neq 0$ in $\mathcal{A}$; this assertion in fact is a restatement of the fact that every two analytic bundles over $\widetilde{R}$ are isomorphic. This fact follows from the Grauert theorem [13]. The function $A \stackrel{\text { def }}{=} \Gamma \circ \varphi$ is $\alpha$-automorphic on $\mathbb{D}$. Since the functions $\left\|\Gamma \circ P^{-1}\right\|,\left\|\Gamma^{-1} \circ P^{-1}\right\|$ are single-valued and continuous on $\bar{R}$, it follows that $A, A^{-1}$ are in $H^{\infty}\left(\mathbb{D}, \mathbb{C}^{k \times k}\right)$.

Proof of Lemma 9.4. Let $A, B$ be the matrix functions that correspond to characters $\alpha, \beta$ as in the above proof of Lemma 9.3, so that $H_{\alpha}^{2}(R)=A H_{\mathbb{I}}^{2}(R), H_{\beta}^{2}(R)=B H_{\mathbb{I}}^{2}(R)$. Let $\Phi: H_{\alpha}^{2}(R) \longrightarrow H_{\beta}^{2}(R)$ be the isometric isomorphism such that $\Phi S_{R, \alpha}=S_{R, \beta} \Phi$. Define a function $\rho$ in $H^{2}\left(\mathbb{D}, \mathbb{C}^{k \times k}\right)$ by $\Phi(A c)=\rho \cdot A c, c \in \mathbb{C}^{n}$. The hypothesis implies that there exists a subdomain $\mathcal{W}$ in $\mathbb{D}$ such that each point in $(z \circ \mathbf{T})(\mathcal{W})$ has only one preimage on $R$. For any $\zeta \in \mathcal{W}$, if $g \in H_{\alpha}^{2}(R)$ and $g(\zeta)=0$, then $g=(z \circ \mathbf{T}-(z \circ \mathbf{T})(\zeta)) h$ for a certain $h \in H_{\alpha}^{2}(R)$, which implies $(\Phi g)(\zeta)=0$. It follows that $\Phi(f)|\mathcal{W}=\rho \cdot f| \mathcal{W}$ for all 
$f \in H_{\alpha}^{2}(R)$. One easily deduces that

$$
\Phi(f)=\rho \cdot f, \quad f \in H_{\alpha}^{2}(R) .
$$

Since $\rho \cdot A$ is $\beta$-automorphic, it follows that

$$
\rho \circ \varphi=\beta(\varphi) \rho \alpha(\varphi)^{-1}, \quad \varphi \in G .
$$

The map $\Phi$ extends to a unitary equivalence of minimal normal extensions [5]. We see from Lemma 9.1 that there exists a unitary operator $\Psi$ on $L^{2}\left(\partial R, \omega, \mathbb{C}^{k}\right)$ such that $\Psi \mid H_{\alpha}^{2}(R)=\Phi$ and $\Psi M_{z(\cdot)}=M_{z(\cdot)} \Psi$. Looking at the action of $\Psi$ on functions $(z(\cdot)-\lambda)^{-1}\left(f \circ \mathbf{T}^{[-1]}\right), f \in H_{\alpha}^{2}(R)$ and bearing in mind (9.3), we conclude that $\Psi f=\left(\rho \circ \mathbf{T}^{[-1]}\right) f$ for all $f \in L^{2}\left(\partial R, \omega, \mathbb{C}^{k}\right)$. Therefore $\rho \circ \mathbf{T}^{[-1]}$ is unitary almost everywhere on $\partial R$, that is, $\rho$ is unitary almost everywhere on $E$. Since $\bigcup_{\varphi \in G} \varphi(E)$ has a full measure in $\partial \mathbb{D}$, it follows that $\rho$ is unitary almost everywhere on $\partial \mathbb{D}$.

We can repeat the whole argument for the operator $\Phi^{-1} f=\rho^{-1}$. $f, f \in H_{\beta}^{2}(R)$. Hence $\rho, \rho^{-1} \in H^{2}\left(\mathbb{D}, \mathbb{C}^{k \times k}\right)$, and $\rho^{*} \rho \equiv I$ on $\partial \mathbb{D}$. Consider $\tilde{\rho}\left(\bar{z}^{-1}\right)=\rho^{*-1}(z), z \in \mathbb{D}$. We conclude that $\rho(z) \equiv u, z \in \mathbb{D}$ for a unitary constant $u \in \mathbf{U}\left(\mathbb{C}^{k}\right)$. Then (9.4) gives $\beta(\varphi)=u \alpha(\varphi) u^{*}$, $\varphi \in G$.

The converse "if" part of the statement is obvious.

Lemma 9.5. Suppose $\Omega$ satisfies (9.1) and $\int\|\Omega\| d \omega<\infty$. Then the set of functions on $\widetilde{R}$ that are holomorphic on $\bar{R}$ is dense in $H^{2}(R ; \Omega)$.

Proof. Let $f \in H^{2}(R ; \Omega)$ and $\varepsilon>0$; then $f \circ \mathbf{T} \in H^{2}(R ; \widetilde{\Omega})$, where $\widetilde{\Omega}=\Omega \circ \mathbf{T}$. We can assume that $\widetilde{R}$ is contained in the double $\widehat{R}$ of $R$ [7]. We make use of the conditional expectation operator $E$ [7]. It is easy to see that $E$ maps $H^{2}(\mathbb{D}, \widetilde{\Omega})$ onto $H^{2}(R, \Omega) \dot{+} N$, where $N$ is a finite-dimensional defect space [7]. If $g \in H^{2}(R ; \Omega)$, then $E(g \circ \mathbf{T})=g$.

Let $\tilde{r}$ be a rational function on $\widehat{\mathbb{C}}$, analytic on $\widehat{\mathbb{C}} \backslash \mathbb{D}$, with $\| \tilde{r}-f \circ$ $\mathbf{T} \|_{H^{2}(R ; \widetilde{\Omega})}<\varepsilon$. Then $E \tilde{r} \in H^{2}(R, \Omega) \dot{+} N$. Put $r$ to be the component of $\tilde{r}$ in $H^{2}(R, \Omega)$. Then $E \tilde{r}$ is meromorphic on $\bar{R}$ and $r$ is analytic on $\bar{R}$. We have $\|E \tilde{r}-f\|_{H^{2}(R ; \Omega)}<\varepsilon$ and $\|r-E \tilde{r}\| \approx \operatorname{dist}\left(E \tilde{r}, H^{2}(R ; \Omega)\right) \leqslant$ $\|E \tilde{r}-f\|<\varepsilon$. Hence $\|r-f\|_{H^{2}(R ; \Omega)}<C \varepsilon$, where $C$ is an absolute constant. 


\section{Characterization of $H_{\mu}^{2}\left(X_{+}\right)$.}

We assume here that the spectral measure of $N$ has no point masses. Then $d \nu=|d z| \mid \gamma_{c}$. We will use the following notation. If $f$ is a function on $\gamma_{c}$ and $g$ its lifting to $\partial \widehat{\Delta}_{+}$, that is, $g((z, \bar{z})) \equiv f(z)$, then we will write $g=f^{\#}, f=g^{\text {b }}$ (note that $z$-projections of different subarcs of $\partial \widehat{\Delta}_{+}$cannot coincide). If $g$ is a function on $\widehat{\Delta}$ or $\widehat{\Delta}_{+}$, we put $g^{b}=\left(g \mid \partial \widehat{\Delta}_{+}\right)^{b}$; in the latter case $g \mid \partial \widehat{\Delta}_{+}$denotes the boundary limit values of $g$. By $(6.3), d e(\cdot)=\mathcal{E}(\cdot)|d z|$, and

$$
\begin{aligned}
\mathcal{E}^{\#}(\delta) & =\frac{1}{2 \pi} \xi(z(\delta))(z(\delta)-\Lambda)^{-1} Q(\delta) \\
& =\frac{1}{2 \pi} \eta(\delta)^{1 / 2}(z(\delta)-\Lambda)^{-1} Q(\delta),
\end{aligned}
$$

for $\delta \in \partial \widehat{\Delta}_{+}$. Since $|\eta| \equiv 1$ on $\partial \widehat{\Delta}_{+}$, the last expression allows us to consider $\mathcal{E}^{\#}$ as a function, defined and meromorphic on a neighbourhood of $\partial \widehat{\Delta}_{+}$in $\widehat{\Delta}$. We use the sets $B, B^{\#}, \operatorname{Pol}_{Q}$ and the natural number $\tilde{\tau}$ that were introduced in Section 7.

\section{Proposition 10.1.}

1) For each $f \in L^{2}(e)$, the equality $f=Q^{b} f$ holds in $L^{2}(e)$.

2) If $\delta \in \partial \widehat{\Delta}_{+} \backslash \operatorname{Pol}_{Q}$ and $m \in M, m=Q(\delta) m$, then $\mathcal{E}(\delta) m=0$ implies $m=0$.

Proof. By (10.1), $\mathcal{E} f=\mathcal{E} Q^{\mathrm{b}} f$, which gives 1 ). Statement 2) also is obvious from (10.1).

Proposition 10.2. The embedding $H_{\mu}^{2}\left(X_{+}\right) \longrightarrow L^{2}(e)$, which is the composition of the isomorphism $a^{-1}: H_{\mu}^{2}\left(X_{+}\right) \longrightarrow E^{2}(\mu)$ with the canonical embedding of $E^{2}(\mu)$ into $L^{2}(e)$, defined by $(6.7)$, is given by $f \in H_{\mu}^{2}\left(X_{+}\right) \longmapsto f^{b}$.

Proof. Let $f=a g, g \in E^{2}(\mu)$, and $h$ be the image of $g$ in $L^{2}(e)$, that is, $g=\mathcal{K}(\cdot-\Lambda) \mathcal{E} h$. By (10.1) and the Plemelj "jump" formula, $g_{i}-g_{e}=Q^{b} h$. Therefore the boundary values of $f$ on $\partial \widehat{\Delta}_{+}$are given by

$$
f^{b}=Q^{b} g_{i}=Q^{b}\left(g_{i}-g_{e}\right)=Q^{b} h \text {. }
$$


By Proposition 10.1, $h=Q^{\mathrm{b}} h=f^{\mathrm{b}}$ in $L^{2}(e)$.

From now on, let us fix points $p_{j}$ on nondegenerate components $\widehat{\Delta}_{j}^{+}$, and let $\omega_{j}$ be the harmonic measure for $\widehat{\Delta}_{j}^{+}$at $p_{j}$. We define the harmonic measure $\omega$ for $\partial \widehat{\Delta}_{+}$by $\omega \mid \partial \widehat{\Delta}_{j}^{+}=\omega_{j}$.

Lemma 10.3. For each $\delta_{0} \in \partial \widehat{\Delta}_{+}$, there exists $c<1$ such that $\|Q(\delta)\| \leqslant\left|z(\delta)-z\left(\delta_{0}\right)\right|^{-c}$ in a neighbourhood of $\delta_{0}$.

Proof. By (10.1), $\left\|\mathcal{E}^{\#}(\delta)\right\| \sim\left|z(\delta)-z\left(\delta_{0}\right)\right|^{-c}$ for some rational $c$. But $\int \mathcal{E}(z)|d z|=I$, which implies that $c<1$. Now we remark that

$$
Q(\delta)=\eta(\delta)^{-1 / 2}(z(\delta)-\Lambda) \mathcal{E}^{\#}(\delta),
$$

which gives the statement of the Lemma.

Definition. Any branching point $\delta$ of $\widehat{\Delta}_{+}$has a neighbourhood $\mathcal{W}$ such that $\mathcal{W} \backslash\{\delta\}$ projects $j$-to-one onto the $z$-plane for some $j \geqslant 2$. We put the order of the branching point $\delta$ to be equal to $j-1$.

Let $\mathrm{Br}$ be the set of branching points of $\widehat{\Delta}_{+}$. Choose $\tau \in \mathbb{N}$ such that the orders of these points do not exceed $\tau$, and $\tau \geq \tilde{\tau}$. Put

$$
\mathcal{P}=\operatorname{Br} \cup\left(\operatorname{Pol}_{Q} \cap \widehat{\Delta}_{+}\right) .
$$

Fix an analytic function $q$ on a neighbourhood of clos $\widehat{\Delta}_{+}$, which has simple zeros in points of $\mathcal{P}$ and no other zeros on clos $\widehat{\Delta}_{+}$.

Let $\widehat{\Delta}_{j}$ be a nondegenerate component of $\widehat{\Delta}$ of multiplicity $k_{j}$. Fix a function $\tilde{X}_{j} \in \operatorname{Hol}\left(\widehat{\Delta}_{j}^{+}, \mathcal{L}\left(\mathbb{C}^{k_{j}}, M\right)\right)$ such that $\tilde{X}_{j}(\delta) \mathbb{C}^{k_{j}}=X(\delta)$ for all $\delta \in \widehat{\Delta}_{j}^{+}$. Since analytic vector bundles $X \mid \widehat{\Delta}_{j}^{+}$are trivial by the Grauert theorem [13], such an $\widetilde{X}_{j}$ exists. Moreover, we will assume $\widetilde{X}_{j}$ to be analytic on a neighbourhood of $\operatorname{clos} \widehat{\Delta}_{j}^{+}$in $\widehat{\Delta}_{j}$. The map $f \longmapsto \widetilde{X}_{j} f$ is an isomorphism between analytic cross-sections of the trivial vector bundle $\widehat{\Delta}_{j}^{+} \times \mathbb{C}^{k_{j}}$ and analytic cross-sections of the bundle $X_{+} \mid \widehat{\Delta}_{j}^{+}$.

Define the weight $\Omega_{j}$ by

$$
\Omega_{j}=\widetilde{X}_{j}^{*} \mathcal{E}^{\#} \tilde{X}_{j} \mid \partial \widehat{\Delta}_{j}^{+} .
$$

We put

$H_{\tau}^{2}\left(\widehat{\Delta}_{j}^{+}, \Omega_{j}\right)=\left\{f \in q^{-\tau} \mathcal{N}^{+}\left(\widehat{\Delta}_{j}^{+} ; \mathbb{C}^{k_{j}}\right):\left(f \mid \partial \widehat{\Delta}_{j}^{+}\right)^{\mathrm{b}} \in L^{2}\left(z\left(\partial \widehat{\Delta}_{j}^{+}\right), \nu, \Omega_{j}\right)\right\}$. 
It follows from (10.1) that $\Omega_{j} \mid \partial \widehat{\Delta}_{j}^{+}$is real analytic, except for a finite number of power-type singularities. The well-known formula

$$
d \omega=-(2 \pi)^{-1} \frac{\partial g}{\partial n} d s,
$$

which expresses the harmonic measure in terms of the Green function [2], [10] implies that the same is true for the function $d \nu^{\# / d \omega}$. By Proposition 10.1.2), $\Omega_{j}$ is invertible in points of $\partial \widehat{\Delta}_{j}^{+} \backslash B^{\#}$. Put

$$
\widetilde{\Omega}_{j}=|q|^{\tau} \frac{d \nu^{\#}}{d \omega} \Omega_{j}
$$

Then there is a scalar function $s$, analytic in a neighbourhood of $\operatorname{clos} \widehat{\Delta}_{j}^{+}$, such that $s \widetilde{\Omega}_{j}$ and $s \widetilde{\Omega}_{j}^{-1}$ are bounded on $\partial \widehat{\Delta}_{+} \cdot$ This implies that $\widetilde{\Omega}_{j}$ meets the log-integrability condition (9.1). Therefore we can rewrite the above definition as

$$
H_{\tau}^{2}\left(\widehat{\Delta}_{j}^{+}, \Omega_{j}\right)=q^{-\tau} H^{2}\left(\widehat{\Delta}_{j}^{+},|q|^{\tau} \frac{d \nu^{\#}}{d \omega} \Omega_{j}\right) .
$$

Functions in this class may have at most poles of order $\tau$ in points of $\mathcal{P}$. by

Let $\left\{\Delta_{j}\right\}$ be all nondegenerate components of $\Delta$. We define $\tilde{X}, \Omega$

$$
\widetilde{X}\left|\widehat{\Delta}_{j}^{+}=\tilde{X}_{j}, \quad \Omega\right| \partial \widehat{\Delta}_{j}^{+}=\Omega_{j},
$$

and put

$$
\operatorname{Hol}\left(\widehat{\Delta}_{+}\right)=\bigoplus_{j} \operatorname{Hol}\left(\widehat{\Delta}_{j}^{+}, \mathbb{C}^{k_{j}}\right), \quad \widetilde{X} H_{\tau}^{2}\left(\widehat{\Delta}_{+}, \Omega\right)=\bigoplus_{j} \widetilde{X}_{j} H_{\tau}^{2}\left(\widehat{\Delta}_{j}^{+}, \Omega_{j}\right) .
$$

We give $\widetilde{X} H_{\tau}^{2}\left(\widehat{\Delta}_{+}, \Omega\right)$ the Hilbert direct sum norm, defined by norms of $H^{2}$-spaces that figure in (10.3).

Theorem 10.4. Suppose that the spectral measure of $N$ has no point masses. Let $\mathcal{K}$ be a compact subset of $\widehat{\Delta}_{+}$such that $\mathcal{P} \subset \operatorname{int} \mathcal{K}, z(\partial \mathcal{K}) \cap$ $z(\mathcal{P})=\varnothing$. Then

$$
H_{\mu}^{2}\left(X_{+}\right)=\left\{f \in \tilde{X} H_{\tau}^{2}\left(\widehat{\Delta}_{+}, \Omega\right):\right.
$$

$$
\left.(f)_{\mathcal{K}}(z) \stackrel{\text { def }}{=} \sum_{\substack{z(\delta)=z \\ \delta \in \mathcal{K}}} f(\delta) \text { is bounded near } z(\mathcal{P})\right\}
$$


and the norms in these two spaces coincide.

Some of the points of $z^{-1}(z(\mathcal{P}))$ may lie on $\partial \widehat{\Delta}_{+}$, but the values of $f$ in neighbourhoods of such points are not taken into account in the above expression for $(f)_{\mathcal{K}}$. Therefore for each $f \in \widetilde{X} H_{\tau}^{2}\left(\widehat{\Delta}_{+}, \Omega\right),(f)_{\mathcal{K}}$ is meromorphic on $z(\mathcal{P})$ and has on it at most poles of order $\tau$. So the above condition on $f$ reduces to a finite number of linear conditions.

Spaces $H_{\mu}^{2}\left(X_{-}\right)$and $\bar{H}_{\mu^{*}}^{2}\left(Y_{ \pm}\right)$can be characterized in the same way.

Denote the right hand part of $(10.4)$ by $\widetilde{H}_{\mu}^{2}\left(X_{+}\right)$.

Proposition 10.5. The map $f \longmapsto f^{b}$ defines isometries of $H_{\mu}^{2}\left(X_{+}\right)$, $\widetilde{H}_{\mu}^{2}\left(X_{+}\right)$into $L^{2}(e)$ (the norm in $\widetilde{H}_{\mu}^{2}\left(X_{+}\right)$is inherited from $H_{\tau}^{2}\left(\widehat{\Delta}_{+}, \Omega\right)$ ).

Proof. The first map is an isometry by Proposition 10.2. The second map is isometric because for $f=\widetilde{X} v \in \widetilde{X} H_{\tau}^{2}\left(\widehat{\Delta}_{+}, \Omega\right)$,

$$
\|f\|_{\widetilde{H}_{\mu}^{2}\left(X_{+}\right)}^{2}=\int\left\langle\Omega^{b} v, v\right\rangle d \nu=\int\langle\mathcal{E} f, f\rangle d \nu=\|f\|_{L^{2}(e)}^{2} .
$$

Proof of TheOrem 10.4. By the above proposition, to prove that $H_{\mu}^{2}\left(X_{+}\right)=\widetilde{H}_{\mu}^{2}\left(X_{+}\right)$, it suffice to check that a complete subset of $H_{\mu}^{2}\left(X_{+}\right)$is contained in $\widetilde{H}_{\mu}^{2}\left(X_{+}\right)$, and vice versa.

1) By (1.11), the functions

$$
\varphi_{t, m}(z)=\frac{\mu(z)}{z-t} m,
$$

with $t \in \mathbb{C} \backslash \gamma$ and $\mu(t) m=0$ are complete in $E^{2}(\mu)$. Therefore the corresponding functions

$$
f(\delta)=\left(a \varphi_{t, m}\right)(\delta)=Q(\delta)(z(\delta)-t)^{-1} m
$$

are complete in $H_{\mu}^{2}\left(X_{+}\right)$. Take any such $f$. Fix a point $z_{0} \in z(\mathcal{P})$, and let us prove that $(f)_{\mathcal{K}}$ does not have pole in $z_{0}$ (the most difficult case is when $z_{0} \in \gamma_{c}$ ). We have

$$
\varphi_{t, m}(z)=a^{-1} f(z)=(f)_{\mathcal{K}}(z)+(f)_{\widehat{\Delta}_{+} \backslash \mathcal{K}}(z), \quad z \in \mathbb{C} \backslash \gamma .
$$

By Lemma 10.3, $\left|(f)_{\widehat{\Delta}_{+} \backslash \mathcal{K}}(z)\right| \leqslant\left|z-z_{0}\right|^{-c}$ in a neighbourhood of $z_{0}$ for some $c<1$. On the other hand, from (6.6) and the fact that 
$(z-\Lambda) \mathcal{E} \in L^{1}(|d z|)$ one can conclude that $\left|\varphi_{t, m}(z)\right|=o\left(\operatorname{dist}\left(z, \gamma_{c}\right)\right)$, $z \longrightarrow z_{0}$. By $(10.5),(f)_{\mathcal{K}}(z)$ cannot have a pole in $z_{0}$.

Let $f=\widetilde{X} g$, then $g$ is meromorphic on a domain containing clos $\widehat{\Delta}_{+}$. Therefore $f \in q^{-N} \oplus \mathcal{N}^{+}\left(\widehat{\Delta}_{j}^{+}, \mathbb{C}^{k_{j}}\right)$. Since

$$
\|g\|_{L^{2}\left(\partial \widehat{\Delta}_{+}, d \nu, \Omega\right)}=\|\left((\cdot-t)^{-1} m \|_{L^{2}(e)}<\infty\right.
$$

we get that $f \in \widetilde{H}_{\mu}^{2}\left(X_{+}\right)$.

2) To prove the converse inclusion, take any $f \in \widetilde{H}_{\mu}^{2}\left(X_{+}\right)$, which has the form $f=\widetilde{X} g$ for a function $g \in q^{-N} \oplus \operatorname{Hol}\left(\widetilde{\Delta}_{j}^{+}, \mathbb{C}^{k_{j}}\right)$, where $\widetilde{\Delta}_{j}^{+}$are neighbourhoods of clos $\widehat{\Delta}_{j}^{+}$. Then, obviously,

$$
\int\left\langle\Omega^{b} g^{b}, g^{b}\right\rangle d \nu=\int\left\langle\mathcal{E} \widetilde{X}^{b} g^{b}, \widetilde{X}^{b} g^{b}\right\rangle d \nu<\infty
$$

By Lemma 9.5, such functions $f=\widetilde{X} g$ are dense in $\widetilde{H}_{\mu}^{2}\left(X_{+}\right)$.

The function $a^{-1} f$ is bounded and analytic on $\mathbb{C} \backslash \gamma_{c}$ and takes value 0 at $\infty$. Writing down its Cauchy representations in the components of $\mathbb{C} \backslash \gamma_{c}$ and summing them up, we arrive at the formula

$$
a^{-1} f=\frac{1}{2 \pi i} \mathcal{K}\left(i \xi f^{b}\right)
$$

because $f^{b}$ is the jump of $a^{-1} f$ on $\gamma_{c}$. Since $f^{b} \in L^{2}(e)$ and $\left(a^{-1} f\right)(\cdot)=$ $\mu(\cdot)\left(a^{-1} f\right)(\cdot)$ on $\mathbb{C} \backslash \gamma_{c}$ (see $\left.(6.1)\right)$, we conclude that $a^{-1} f \in E^{2}(\mu)$. Thus $f \in a E^{2}(\mu)=H_{\mu}^{2}\left(X_{+}\right)$.

\section{Simple subnormal operators.}

Definitions. A polynomial $P(z, w)$ will be called admissible if it has a form

$$
P(z, w)=\sum_{i=0}^{n} \sum_{j=0}^{n} a_{i j} z^{i} w^{j},
$$

where $n>0$ is an integer, $a_{n n}=1$, and $a_{j i}=\bar{a}_{i j}, 0 \leqslant i, j \leqslant n$, and $P$ has no irreducible factors of the type $z-z_{0}, w-w_{0}$. An algebraic curve $\Delta$ in $\mathbb{C} P^{2}$ is called admissible if it is given by an equation $P(z, w)=0$ for some admissible $P$. We put rank $\Delta=n$. 
If $\Delta=\Delta_{1}^{k_{1}} \cup \cdots \cup \Delta_{T}^{k_{T}}$ is the decomposition of $\Delta$ into the union of irreducible curves, then $\operatorname{rank} \Delta=\sum k_{j} \operatorname{rank} \Delta_{j}$.

One sees from (0.1) that each discriminant curve of the type studied above without degenerate components is admissible. Also, the product of two admissible polynomials is admissible.

Lemma 11.1. For each admissible algebraic curve $\Delta$, the sets $z\left(\widehat{\Delta}_{+}\right)$ and $w\left(\widehat{\Delta}_{-}\right)$are bounded.

ProOF. Let $w=w_{k}(z)$ be all (possibly multivalued) analytic functions that are defined in a neighbourhood of $z=\infty$ by the implicit equation $P(z, w)=0$. Put $K=\sum_{i, j}\left|a_{i j}\right|$. Then $(z, w) \in \Delta$ and $|z|>K$ imply $|w| \leqslant K$ (by the triangle inequality). It follows that

$$
w_{k}(z)=\lambda_{k}+\rho_{k} \varphi_{k}\left(z^{-1}\right)^{c_{k}}
$$

where $\lambda_{k}, \rho_{k} \in \mathbb{C}, c_{k}>0$ are rational and $\varphi_{k}$ are analytic in 0 , with $\varphi_{k}(0)=0$ and $\varphi_{k}^{\prime}(0)=1$. An easy calculus now shows that

$$
-\eta((z, w))^{-1}=\frac{d w}{d z} \longrightarrow 0
$$

if $w=w_{k}(z)$ and $z \longrightarrow \infty$. This shows that $z\left(\widehat{\Delta}_{+}\right)$is bounded. The set $w\left(\widehat{\Delta}_{-}\right)$is also bounded, because $w\left(\widehat{\Delta}_{-}\right)=\overline{z\left(\Delta_{+}\right)}$.

Now let $\widehat{\Delta}$ be the discriminant surface of a subnormal $S$ of finite type such that the spectral measure of $N$ has no point masses (but we allow $\widehat{\Delta}$ to have degenerate components). As before, let $\widehat{\Delta}_{j}$ be the nondegenerate components of $\widehat{\Delta}$, pick points $p_{j} \in \widehat{\Delta}_{j}^{+}$and denote by $\omega_{j}$ the harmonic measures of $\widehat{\Delta}_{j}^{+}$with respect to $p_{j}$. Put $\omega=\sum_{j} \omega_{j}$. The corresponding measure $\omega^{b}$ on $\gamma_{c}$ is absolutely continuous with respect to the arc length measure.

We can define an $H^{\infty}\left(\widehat{\Delta}_{+}\right)$-calculus for $N$ : we put

$$
f(N)=\left(f \mid \widehat{\Delta}_{+}\right)^{\mathrm{b}}(N), \quad f \in H^{\infty}\left(\widehat{\Delta}_{+}\right) .
$$

Definition. Let $N$ have no point masses. Then $S$ is called simple if it admits the $H^{\infty}\left(\widehat{\Delta}_{+}\right)$-calculus, that is, $f(N) H \subset H$ for all $f \in$ $H^{\infty}\left(\widehat{\Delta}_{+}\right)$. 
Choose a function $r$, which is holomorphic on a neighbourhood of clos $\widehat{\Delta}_{+}$and has zeros exactly in branching points of $\widehat{\Delta}_{+}$of orders that are equal to orders of these points. Let $\mathcal{K}$ be the subset of $\widehat{\Delta}_{+}$from Theorem 10.4.

\section{Proposition 11.2.}

i) For any $f \in \operatorname{Hol}\left(\widehat{\Delta}_{+}\right),\left(r^{-1} f\right)_{\mathcal{K}}$ is bounded near $z(\mathcal{P})$.

ii) If $f$ is meromorphic on $\widehat{\Delta}_{+}$and $(\varphi f)_{\mathcal{K}}$ is bounded near $z(\mathcal{P})$ for all $\varphi \in H^{\infty}\left(\widehat{\Delta}_{+}\right)$, then $r \in \operatorname{Hol}\left(\widehat{\Delta}_{+}\right)$.

The proof of i) is elementary. When proving ii), one can use the fact that if $\delta_{0}, \ldots, \delta_{l}$ are points of $\widehat{\Delta}_{+}$that project into the same point $z_{0}$ and $\mathcal{W}_{0}, \ldots, \mathcal{W}_{l}$ are their small neighbourhoods and $\psi$ is analytic in $\mathcal{W}_{0}$, then for any $s \in \mathbb{N}$ there is $\varphi$ in $H^{\infty}\left(\widehat{\Delta}_{+}\right)$such that $\varphi-\psi \mid \mathcal{W}_{0}$ and $\varphi \mid \mathcal{W}_{j}, j \geqslant 1$ have zeros of orders $\geqslant s$ in $\delta_{j}$. We omit the details.

Note that Proposition 11.2 is true for vector-valued functions $f \in$ $\operatorname{Hol}\left(\widehat{\Delta}_{+}, M\right)$ as well.

Proposition 11.3. The following are equivalent.

1) $S$ is simple.

2) $r Q$ is analytic on $\widehat{\Delta}_{+}$.

3) $H_{\mu}^{2}\left(X_{+}\right)=r^{-1} \tilde{X} H^{2}\left(\widehat{\Delta}_{+}, \Omega^{\prime}\right)$, where

$$
\Omega^{\prime}=|r| \frac{d \nu^{\#}}{d \omega} \Omega
$$

Proof. 2) implies 1). If 2) holds, then for every $f \in H_{\mu}^{2}\left(X_{+}\right), r f$ is analytic on $\widehat{\Delta}_{+}$(because functions $Q(\cdot)(z(\cdot)-t)^{-1} m$ with $m \in M$, $t \in \mathbb{C} \backslash \gamma, \mu(t) m=0$ are complete in $\left.H_{\mu}^{2}\left(X_{+}\right)\right)$. By Proposition 11.2.i) and (10.4), $H^{\infty}\left(\widehat{\Delta}_{+}\right)$acts by multiplication on $H_{\mu}^{2}\left(X_{+}\right)$. Therefore $S$ is simple.

1) implies 3). By Proposition 11.2.i) and (10.4),

$$
r^{-1} \widetilde{X} H^{2}\left(\widehat{\Delta}_{+}, \Omega^{\prime}\right) \subset H_{\mu}^{2}\left(X_{+}\right)
$$


If $f \in H_{\mu}^{2}\left(X_{+}\right)$, then $(\varphi f)_{\mathcal{K}}$ has to be bounded near $z(\mathcal{P})$ for all $\varphi \in$ $H^{\infty}\left(\widehat{\Delta}_{+}\right)$. By Proposition 11.2.ii $), f$ is in $r^{-1} \operatorname{Hol}\left(\widehat{\Delta}_{+}\right)$, and so by Theorem 10.4, $f \in r^{-1} \widetilde{X} H^{2}\left(\widehat{\Delta}_{+}, \Omega^{\prime}\right)$.

3) implies 2). Trivially, because $Q(\cdot) m \in H_{\mu}^{2}\left(X_{+}\right)$for all $m \in M$.

These arguments imply that for a simple subnormal $S$, the order of pole of $Q$ at each branching point of $\widehat{\Delta}_{+}$equals to the order of this branching point.

We remark that if 1)-3) hold, then the range norm on

$$
r^{-1} \tilde{X} H^{2}\left(\widehat{\Delta}_{+},|r| \frac{d \nu^{\#}}{d \omega} \Omega\right)
$$

coincides with the norm on $H_{\mu}^{2}\left(X_{+}\right)$.

The following lemma extends a result by McCarthy and Yang [16, Theorem 1.12].

Lemma 11.4. Let $\Delta$ be any irreducible admissible separated algebraic curve, and $\Omega$ a matrix $\mathbb{C}^{k \times k}$-valued log-integrable weight. Define an operator $S$ on $H^{2}\left(\widehat{\Delta}_{+}, \Omega\right)$ by $S f(\delta)=z(\delta) f(\delta)$ (to define the harmonic measure, we choose any base point). Then $S$ is a simple subnormal operator of finite type and $\operatorname{rank}\left[S^{*}, S\right]=k \operatorname{rank} \Delta$.

Proof. Lemma 11.1 implies that $S$ is bounded. By Lemmas 9.1 and 9.2, it is subnormal, and its minimal normal extension is given by $N f(\delta)=z(\delta) f(\delta), f \in L^{2}\left(\partial \widehat{\Delta}_{+}, \omega, \Omega\right)$. The operator $S^{*}$ acts by

$$
S^{*} f=P_{H^{2}\left(\widehat{\Delta}_{+}, \Omega\right)}(\bar{z} f), \quad f \in H^{2}\left(\widehat{\Delta}_{+}, \Omega\right),
$$

which gives

$$
\begin{aligned}
\operatorname{Ker}\left[S^{*}, S\right] & =\left\{f:\|S f\|=\left\|S^{*} f\right\|\right\} \\
& =\left\{f \in H^{2}\left(\widehat{\Delta}_{+}, \Omega\right): \bar{z} f \in H^{2}\left(\widehat{\Delta}_{+}, \Omega\right)\right\} \\
& =\left\{f \in H^{2}\left(\widehat{\Delta}_{+}, \Omega\right): w(\cdot) f(\cdot) \in \operatorname{Hol}\left(\widehat{\Delta}_{+}, \mathbb{C}^{k}\right)\right\} .
\end{aligned}
$$

The sum of orders of all poles of $w(\cdot)$ on $\widehat{\Delta}$ equals rank $\Delta$. By Lemma $11.1, w(\cdot)$ has no poles on $\widehat{\Delta}_{-}$. Hence the last expression in (11.2) gives $\operatorname{Codim} \operatorname{Ker}\left[S^{*}, S\right]=k \operatorname{rank} \Delta$. By the remark in [25, Section 6], the 
nondegenerate part of the discriminant surface of $S$ coincides with $\Delta$. As $S$ trivially admits $H^{\infty}\left(\widehat{\Delta}_{+}\right)$-calculus, it is simple.

Xia's exposition in [23] uses the notion of a "quadrature domain on a Riemann surface". He defines it as a domain $\mathcal{D}$ with a boundary, consisting of rectifiable Jordan curves on a branched Riemann surface equipped with a projection $z(\cdot)$ onto $\mathbb{C}$ such that $w(\cdot)=\overline{z(\cdot)}$ for some function $w(\cdot)$, meromorphic on $\operatorname{clos} \mathcal{D}$. It is easy to see that $(\mathcal{D}, z(\cdot))$ is a quadrature domain on a Riemann surface if and only if $\mathcal{D}=\widehat{\Delta}_{+}$for some irreducible admissible separated algebraic curve $\widehat{\Delta}$ in $\mathbb{C}^{2}$.

Theorem 11.5. A Hilbert space operator is simple subnormal of finite type if and only if there exist an admissible separated algebraic curve $\Delta=\cup \Delta_{j}^{k_{j}}$, points $p_{j} \in \widehat{\Delta}_{j}^{+}$and homomorphisms $\alpha_{j} \in \operatorname{Char}_{k_{j}}\left(\widehat{\Delta}_{j}^{+}\right)$ such that $S$ is unitarily equivalent to

$$
\bigoplus_{j} S_{\Delta_{j}^{+}, \alpha_{j}, p_{j}}
$$

(see the notation in Section 9). In this case, $\operatorname{rank}\left[S^{*}, S\right]=\operatorname{rank} \Delta$.

Proof. If $S$ is simple subnormal, then by Proposition 11.3, $S$ is unitarily equivalent to the operator of multiplication by $z(\cdot)$ on the space

$$
H^{2}\left(\widehat{\Delta}_{+}, \Omega^{\prime}\right)=\bigoplus_{j} H^{2}\left(\widehat{\Delta}_{j}^{+}, \Omega_{j}^{\prime}\right)
$$

where $\Delta$ is the discriminant curve of $S$ and $\Delta_{j}$ are its nondegenerate components. Then $\cup \Delta_{j}^{k_{j}}$ is an admissible separated algebraic curve. By Lemma 9.1, $S$ is unitarily equivalent to an operator of the form (11.3).

The converse follows from Lemma 11.4.

REMARK. The discriminant curve of a simple subnormal operator has no degenerate components.

Indeed, let $S$ be simple, and let $\Delta=\bigcup \Delta_{j}^{k_{j}} \cup \bigcup \Delta_{l}^{\prime c_{l}}$ be the the discriminant curve of $S$, where the $\Delta_{l}$ are nondegenerate components of $\Delta$ and $\Delta_{l}^{\prime}$ are degenerate. Put $n=\operatorname{rank} \Delta$. By Lemma 11.4,

$$
\sum_{j} k_{j} \operatorname{rank} \Delta_{j}+\sum_{l} c_{l}=n=\operatorname{rank}\left[S^{*}, S\right]=\sum_{j} k_{j} \operatorname{rank} \Delta_{j} .
$$


The following statement answers the questions of simiarity and unitary equivalence of simple subnormal operators.

\section{Proposition 11.6.}

1) Two simple subnormal operators are similar if and only if their discriminant surfaces coincide and the multiplicities of their irreducible components also agree.

2) Let $S=\oplus_{j} S_{\widehat{\Delta}_{j}^{+}, \alpha_{j}, p_{j}}$ and $S^{\prime}=\oplus_{j} S_{\widehat{\Delta}_{j}^{+}, \alpha_{j}^{\prime}, p_{j}}$ be two simple subnormal operators with the same discriminant curve $\Delta=\cup \Delta_{j}^{k_{j}}$ and the same choice of base points $p_{j} \in \widehat{\Delta}_{j}^{+}$. Then $S$ and $S^{\prime}$ are unitarily equivalent if and only if $\alpha_{j} \sim \alpha_{j}^{\prime}$ for all $j$.

Proof. 1). The "if" part follows from Theorem 11.5 and Corollary to Lemma 9.3. The converse follows from the Remark in [25, Section 6].

Let $S, S^{\prime}$ be as in 2), and let $S^{\prime}=L^{-1} S L$, where $L$ is a unitary isomorphism. Then $f\left(S^{\prime}\right)=L^{-1} f(S) L$ for all $f \in H^{\infty}\left(\widehat{\Delta}_{+}\right)$, which implies that $L$ splits: $L=\oplus L_{j}$, where for each $j, L_{j}$ is a unitary isomorphism between $H_{\alpha_{j}}^{2}\left(\widehat{\Delta}_{j}^{+}\right)$and $H_{\alpha_{j}^{\prime}}^{2}\left(\widehat{\Delta}_{j}^{+}\right)$. Now the statement follows from Lemma 9.4.

Let $S$ be rationally cyclic and irreducible. It is easy to see that then $\widehat{\Delta}$ has only one nondegenerate component $\widehat{\Delta}_{1}, \widehat{\Delta}_{1}^{+}$projects homeomorphically into $\mathbb{C}$ and $k_{1}=1$. For this case, the above fact has been proved by McCarthy and Yang ([16, Theorem 2.2]).

\section{Internal Riemann surface models and general structure theorems.}

\subsection{Elimination of point masses.}

Since $N$ is unitarily equivalent to the operator of multiplication by $z$ on $L^{2}(e), S$ has no point masses if and only if $e(\cdot)$ is absolutely continuous with respect to the arc length measure. In general, as follows from Theorem A, $e(\cdot)=e_{a}(\cdot)+e_{s}(\cdot)$, where $e_{a}$ is absolutely continuous and $e_{s}$ is a finite sum of matrix point masses. Since $N$ is unitarily equivalent to $M_{z}$ on $L^{2}(e)$, we have the corresponding orthogonal sum 
decompositions

$$
K=K_{a} \oplus K_{s}, \quad N=N_{a} \oplus N_{s}
$$

Put $H_{0}=P_{K_{a}} H$ and consider an operator $L: H \rightarrow H_{0}$, given by

$$
L x=P_{K_{a}} x, \quad x \in H .
$$

The following simple fact will be used in the sequel: if $T$ is a subnormal operator and $T_{1}$ its restriction to an invariant subspace of finite codimension, then $\operatorname{rank}\left[T^{*}, T\right]<\infty$ if and only if $\operatorname{rank}\left[T_{1}^{*}, T_{1}\right]<\infty$.

Lemma 12.1. The space $H_{0}$ is closed and $L$ is invertible. The operator $S_{0} \stackrel{\text { def }}{=} L S L^{-1}$ on $H_{0}$ is pure subnormal without point masses, and $\operatorname{rank}\left[S_{0}^{*}, S_{0}\right]<\infty$.

Proof. We have

$$
\langle L x, L x\rangle=\left\langle\left(I-P_{H} P_{K_{s}} P_{H}\right) x, x\right\rangle, \quad x \in H .
$$

Let $t$ be the maximal eigenvalue of the finite rank self-adjoint operator $P_{H} P_{K_{s}} P_{H}$. Since $H \cap K_{s}=0$, it follows that $L$ is one-to-one, and therefore $t<1$. Hence $L$ is an isomorphism onto its range. Obviously, $L S x=L N x=N L x$ for $x \in H$, which implies that $N \mid K_{a}$ is a normal extension of $S_{0}$ with absolutely continuous spectrum. So $S_{0}$ has no point masses.

At last, put $H_{1}=H \cap K_{a}$ and $S_{1}=S \mid H_{1}$. Since rank $H \ominus H_{1}<\infty$, we have $\operatorname{rank} H_{0} \ominus H_{1}<\infty$, and thus $\operatorname{rank}\left[S_{1}^{*}, S_{1}\right]<\infty, \operatorname{rank}\left[S_{0}^{*}, S_{0}\right]<$ $\infty$.

So for any operator $S$ we have defined in a canonical way a subnormal operator $S_{0}$ without point masses, which is similar to $S$.

\subsection{Passage to a simple subnormal operator: a finite-dimen- sional extension.}

Here we assume $S$ to be a subnormal operator of finite type without point masses. Define a linear manifold

$$
\widetilde{H}=\operatorname{span}\left\{f(N) H: f \in H^{\infty}\left(\widehat{\Delta}_{+}\right)\right\},
$$


clearly, $\widetilde{H} \supset H$.

\section{Proposition 12.2.}

1) $\widetilde{H}$ is a closed invariant subspace of $N$, and $\operatorname{dim} \widetilde{H} \ominus H$ is finite.

2) $\widetilde{S} \stackrel{\text { def }}{=} N \mid \widetilde{H}$ is a simple subnormal operator.

Proof. Let $f \in H^{\infty}\left(\widehat{\Delta}_{+}\right)$, and let $\widetilde{U}, W$ be the operators from Section 6.2. By Theorem 10.4, the multiplication by $f$ sends $H_{\mu}^{2}\left(X_{+}\right)$into $\widetilde{X} H_{\tau}^{2}\left(\widehat{\Delta}_{+}, \Omega\right)$. This action agrees with the action of $f(N)$, that is, if $x \in H$ and $u=a U x \in H_{\mu}^{2}\left(X_{+}\right)$, then

$$
f^{b} u^{b}=W f(N) x
$$

(see Proposition 10.2). Let

$$
\mathcal{H}\left(X_{+}\right)=\operatorname{span}\left\{f u: f \in H^{\infty}\left(\widehat{\Delta}_{+}\right), u \in H_{\mu}^{2}\left(X_{+}\right)\right\},
$$

it follows from (12.1), (12.2) that

$$
\widetilde{H}=\left\{W^{-1} u^{b}: u \in \mathcal{H}\left(X_{+}\right)\right\} .
$$

Since $H_{\mu}^{2}\left(X_{+}\right) \subset \mathcal{H}\left(X_{+}\right) \subset \widetilde{X} H_{\tau}^{2}\left(\widehat{\Delta}_{+}, \Omega\right), \mathcal{H}\left(X_{+}\right)$is closed and

$$
\operatorname{dim} H_{\mu}^{2}\left(X_{+}\right) \ominus \mathcal{H}\left(X_{+}\right)<\infty
$$

By Proposition 10.2, the map $u \longmapsto W^{-1} u^{b}$ is an isometry from

$$
\widetilde{X} H_{\tau}^{2}\left(\widehat{\Delta}_{+}, \Omega\right)
$$

into $L^{2}(e)$. This implies both assertions of 1$)$. Since $N$ is a normal extension of $\widetilde{S}$ and $f \cdot \mathcal{H}\left(X_{+}\right) \subset \mathcal{H}\left(X_{+}\right)$for all $f \in H^{\infty}\left(\widehat{\Delta}_{+}\right)$, we obtain $2)$.

It is clear that $\widetilde{H}$ is the minimal closed subspace of $K$ such that $\widetilde{H} \supset H$ and $N \mid \widetilde{H}$ is simple subnormal. 


\subsection{Main structure result.}

Let $S$ be a subnormal operator of finite type. Following the above procedures, we can construct from it an operator $S_{0}$ without point masses and then a simple operator $\widetilde{S}_{0}$. The operator $\widetilde{S}_{0}$ may be called the canonical simple operator that corresponds to $S$.

Theorem 12.3. Let $S$ be a subnormal operator of finite type. Let $S_{0}$ be the operator obtained from $S$ by eliminating point masses, and $\widetilde{S}_{0}$ be the canonical simple operator corresponding to $S_{0}$; suppose that $\widetilde{S}_{0}$ acts on a space $\widetilde{H}_{0}$. Then

1) There exist eigenvalues $\bar{\lambda}_{k}, 1 \leq k \leq r$ of $\widetilde{S}_{0}^{*}$ and corresponding Jordan chains $\left\{\psi_{\lambda_{k}}^{j}\right\}_{j=0}^{m_{k}}$ of generalized eigenvectors: $\left(\widetilde{S}_{0}^{*}-\bar{\lambda}_{k}\right) \psi_{\lambda_{k}}^{0}=0$, $\left(\widetilde{S}_{0}^{*}-\bar{\lambda}_{k}\right) \psi_{\lambda_{k}}^{j}=\psi_{\lambda_{k}}^{j-1}, j=1, \ldots, m_{k}$, such that $S_{0}=\widetilde{S}_{0} \mid H_{0}$, where

$$
H_{0}=\left\{x \in \widetilde{H}_{0}:\left\langle x, \psi_{\lambda_{k}}^{j}\right\rangle=0, \quad 1 \leqslant k \leqslant r, 0 \leqslant j \leqslant m_{k}\right\}
$$

(the $\lambda_{k}$ 's are not necessarily distinct).

2) There is a finite set $\left\{\mu_{j}\right\}$ and operators $l_{j}: H_{0} \longrightarrow \mathbb{C}^{t_{j}}, t_{j} \in \mathbb{N}$, $1 \leqslant j \leqslant m$, with $\left(S_{0}^{*}-\bar{\mu}_{j}\right) l_{j}^{*}=0$ such that the operator $S$ coincides with $S_{0}$, acting on the renormed space $\left(H_{0},\|\cdot\|_{1}\right)$, where

$$
\|x\|_{1}^{2} \stackrel{\text { def }}{=}\|x\|^{2}+\sum_{j=1}^{m}\left\|l_{j} x\right\|^{2} .
$$

Conversely, let $\widetilde{S}_{0}$ be any simple subnormal operator of finite type and let $S$ be obtained from $\widetilde{S}_{0}$ by applying the above procedure, where $\left\{\psi_{\lambda_{j}}^{j}\right\}$ and $\left\{l_{j}\right\}$ are arbitrary finite families with the above properties. Then $S$ is a pure subnormal of finite type.

The first part of this theorem is a corrected version of Xia's Theorem 3 in [23]. Xia's formulation is not accurate, because it would follow from it that every subnormal operator of finite type is simple (see example 1) below).

If one combines this theorem with Theorem 11.5, he will obtain a functional model representation of an arbitrary subnormal operator of finite type. One can call it the internal Riemann surface representation. 
REMARK. If $S$ is obtained from $\widetilde{S}_{0}$ in the way described in the theorem and $S^{\prime}$ is the canonical simple subnormal that corresponds to $S$, then $S^{\prime}$ may be different from $\widetilde{S}_{0}$. For instance, if $\widetilde{S}_{0}$ is the standard shift operator $\widetilde{S}_{0} f(z)=z f(z)$ on $H^{2}$, then each its restriction $S=S_{0}$ to an invariant subspace of finite codimension is simple itself, so that $S^{\prime}=S$ and $S^{\prime} \neq \widetilde{S}_{0}$.

ExAmPles. 1) Let $S=M_{z} \oplus M_{z}$ on $H=H^{2}(\mathbb{D}) \oplus H^{2}(\mathbb{D}+1 / 2)$, then $S$ is a simple subnormal operator with $\operatorname{rank}\left[S^{*}, S\right]=2$. Put

$$
\langle(f, g), \psi\rangle=f\left(\frac{3}{4}\right)-g\left(\frac{3}{4}\right), \quad(f, g) \in H .
$$

It is immediate that $\left(S^{*}-3 / 4\right) \psi=0$, so that

$$
H_{0}=(\psi)^{\perp}=\left\{(f, g): f\left(\frac{3}{4}\right)=g\left(\frac{3}{4}\right)\right\}
$$

is an invariant subspace of $S$, and $S_{0}=S \mid H_{0}$ is a subnormal of finite type. The discriminant curve of $S$ is

$$
\Delta=\{z w=1\} \bigcup\left\{\left(z-\frac{1}{2}\right) w=1\right\}
$$

which implies that $\Delta_{+}$, as a Riemann surface over the $z$-plane, consists of two sheaves that cover in a bijective way, respectively, $\mathbb{D}$ and $\mathbb{D}+1 / 2$. Since $H_{0}$ is not $H^{\infty}\left(\widehat{\Delta}_{+}\right)$-invariant, $S_{0}$ is not simple. The canonical simple subnormal operator that corresponds to $S_{0}$ coincides with $S$. One can say that $S_{0}$ is obtained from $S$ by "glueing" the points of $\Delta_{+}$ over $3 / 4$.

2 ) Suppose $S$ is a simple subnormal operator, $\widehat{\Delta}$ has only one nondegenerate component of multiplicity one, and $\widehat{\Delta}_{+}$is simply connected. By Theorem 11.5, $S$ is unitarily equivalent to the multiplication operator $M_{z}$ on $H^{2}\left(\widehat{\Delta}_{+}\right)$(for any base point in $\widehat{\Delta}_{+}$), and let us identify $S$ with this model. Suppose $\widehat{\Delta}_{+}$has a branching point $\delta_{0}$ of order 1 . For instance, one can take

$$
S f(\zeta)=\left(\zeta+\frac{1}{2}\right)^{2} f(\zeta)
$$

on $H^{2}$. Then $\widehat{\Delta}_{+}$can be identified with the unit disc, with the $z$ projection given by $z(\zeta)=(\zeta+1 / 2)^{2}, \zeta \in \mathbb{D}$. Fix a branch of the 
function $\psi(\delta)=\left(z(\delta)-z\left(\delta_{0}\right)\right)^{1 / 2}$, then $\psi$ is an analytic homeomorphism of a neighbourhood of $\delta_{0}$ onto a neighbourhood of 0 . Put

$$
l u=\left(u \circ \psi^{-1}\right)^{\prime}(0), \quad u \in H^{2}\left(\widehat{\Delta}_{+}\right)
$$

Then $l\left(S-z\left(\delta_{0}\right)\right)=l M_{\psi^{2}}=0$, so that we can put $\|u\|_{1}^{2}=\|u\|^{2}+|l u|^{2}$, $u \in H^{2}\left(\widehat{\Delta}_{+}\right)$(see (12.3)). This example shows that the expression for $\left\|l_{j} x\right\|^{2}$ in (12.3) may fail to have the form

$$
\sum_{z\left(\delta_{s}\right)=z\left(\delta_{t}\right)=\mu}\left\langle e_{s t} u\left(\delta_{s}\right), u\left(\delta_{t}\right)\right\rangle,
$$

which was given in $[23$, Theorem 3$]$.

Proof of TheOrem 12.3. 2) We use the notation of Section 12.1. Put $\|x\|_{1}=\left\|L^{-1} x\right\|, x \in H_{0}$. By the spectral theorem, there exist $t_{j} \in \mathbb{N}, G_{j}: K \longrightarrow \mathbb{C}^{t_{j}}$ and $\mu_{j} \in \mathbb{C}$ such that $G_{j} N y=\mu_{j} y$ and $\left\|P_{K_{s}} y\right\|^{2}=\sum_{1}^{r}\left\|G_{j} y\right\|^{2}$ for all $y$ in $K$. Put $l_{j}=G_{j} L^{-1}$. Then

$$
\begin{gathered}
l_{j}\left(S_{0}-\mu_{j}\right)=G_{j}\left(S-\mu_{j}\right) L^{-1}=G_{j}\left(N-\mu_{j}\right) L^{-1}=0 \\
\|x\|_{1}^{2}=\|x\|^{2}+\left\|P_{K_{s}} L^{-1} x\right\|^{2}=\|x\|^{2}+\sum_{j=1}^{r}\left\|l_{j} x\right\|^{2}, \quad x \in H_{0} .
\end{gathered}
$$

This proves the statement.

1) By Section $12.2, S_{0}$ is a restriction of $\widetilde{S}_{0}$ to its invariant subspace $H_{0}$ such that $\operatorname{dim} \widetilde{H}_{0} \ominus H_{0}$ is finite. Put $R=\widetilde{H}_{0} \ominus H_{0}$, then $\widetilde{S}_{0}^{*} R \subset R$. So the statement follows from the linear algebra theorem on the Jordan structure, applied to the operator $\widetilde{S}_{0}^{*} \mid R$.

Let $S^{1}, S^{2}$ be two subnormals of finite type. Let $S_{0}^{1}, S_{0}^{2}$ be the corresponding subnormals without point masses and $\widetilde{S}_{0}^{1}, \widetilde{S}_{0}^{2}$ the corresponding simple subnormals. Then $S^{1}, S^{2}$ are unitarily equivalent if and only if $\widetilde{S}_{0}^{1}, \widetilde{S}_{0}^{2}$ are unitarily equivalent, $S_{0}^{1}, S_{0}^{2}$ are obtained from $\widetilde{S}_{0}^{1}, \widetilde{S}_{0}^{2}$ by passing to the same invariant subspace (in the sense of the latter unitary equivalence), and $S^{1}, S^{2}$ are obtained from $S_{0}^{1}, S_{0}^{2}$ by the same finite rank perturbation of the norm. We remind the reader that the question of unitary equivalence of simple subnormals has been completely answered in Proposition 11.6. 
Notation (some of the entries appear several times).

$M, C, \Delta, \Lambda$

Section 0

$F, e, \mathcal{E}, G, E^{2}(\mu), E_{0}^{2}(1-\mu), \bar{E}^{2}\left(\mu^{*}\right), \bar{E}_{0}^{2}\left(1-\mu^{*}\right), L^{2}(e)$,

$L(z), L^{\prime}(z), \mathcal{K}, \overline{\mathcal{K}}, \mathcal{K} F \mathcal{E} L^{2}(e), \overline{\mathcal{K}} G^{*} \mathcal{E} L^{2}(e), P_{\mu}, \varphi_{t, m}, \varphi_{*, t, m}$,

$\mu(z),\langle\cdot, \cdot\rangle_{d}$

Section 1

$A, H, H^{\prime}, H_{*}, H_{*}^{\prime}, K, M, M_{*}, L(z), L^{\prime}(z), N, P_{H}, P_{H^{\prime}}$,

$\widetilde{U}, \widetilde{V}, \gamma, \mu(z), \rho(z)$

Section 2

$W$

Section 3

$J, H(z), U, V$

Section 4

$Q, T, z(\cdot), w(\cdot), \gamma, \gamma_{c}, \widehat{\Delta}_{j}, \widehat{\Delta}_{+}, \widehat{\Delta}_{-}, \widehat{\Delta}^{\prime}, \delta^{*}, \widehat{\Delta}_{\mathrm{deg}}, \widehat{\Delta}_{\mathrm{ndeg}}$,

$\sigma_{C}(\Lambda), \eta(z), \mu(z), \xi(z)$

Section 6.1

$e, \mathcal{K}(\cdot-\Lambda) \mathcal{E} L^{2}(e), \overline{\mathcal{K}} \mathcal{E} L^{2}(e), j, S^{\prime}, W$

Section 6.2

$a, b, B, H_{\mu}^{2}\left(X_{+}\right), H_{\mu}^{2}\left(X_{-}\right), \bar{H}_{\mu^{*}}^{2}\left(Y_{+}\right), \bar{H}_{\mu^{*}}^{2}\left(Y_{-}\right), \mathrm{Pol}_{Q}$,

$X, X_{ \pm}, Y, Y_{ \pm}, \tilde{\tau}$

Section 7

$\alpha_{1}, \alpha_{2}$

Section 8

$\operatorname{Char}_{k}(R), H_{\alpha}^{2}(R), H^{2}(R, \Omega), L^{2}(\partial R, \omega, \Omega), \operatorname{Möb}(\mathbb{D})$,

$\mathcal{N}(R), \mathcal{N}^{+}(R), S_{R, \alpha}, S_{R, \alpha, p}, \mathbf{T}, \mathbf{U}\left(\mathbb{C}^{k}\right)$

Section 9

$\mathrm{Br}, \mathcal{E}^{\#}, q, Q^{b}, \mathcal{P}, H_{\tau}^{2}\left(\widehat{\Delta}_{j}^{+}, \Omega_{j}\right), H_{\tau}^{2}\left(\widehat{\Delta}_{+}, \Omega\right), \widetilde{X}_{j}, \widetilde{X}$,

$\tau, \omega_{j}, \omega, \Omega_{j}, \Omega$

Section 10

$\operatorname{rank} \Delta, r, \Omega^{\prime}$

Section 11

$H_{0}, H_{1}, K_{a}, K_{s}, L$

Section 12

Acknowledgements. The author is grateful to S. Fedorov and M. Putinar for useful discussions.

\section{References.}

[1] Abrahamse, M. B., Douglas, R. G., A class of subnormal operators related to multiply-connected domains. Advances in Math. 19 (1976), 106-148. 
[2] Ahern, P. R., Sarason, D., The $H^{p}$ spaces of a class of function algebras. Acta Math. 117 (1967), 123-163.

[3] Alpay, D., Vinnikov, V., Analogues d'espaces de de Branges sur des surfaces de Riemann. C. R. Acad. Sci. Paris sér I Math. 318 (1994), 1077-1082.

[4] Baumgartel, H., Analytic Perturbation Theory for Matrices and Operators. Birkhäuser, 1985.

[5] Conway, J. B., The Theory of Subnormal Operators. American Math. Society, 1991. (Math. Surveys and Monographs, vol. 36)

[6] Duren, P. L., Theory of $H^{p}$-spaces. Academic Press, 1970.

[7] Earle, C. J., Marden, A., On Poincaré series with application to $H^{p}$ spaces on bordered Riemann surfaces. Illinois J. Math. 13 (1969), 202219.

[8] Fedorov, S., Harmonic Analysis in a Multiply-Connected Domain. I, II. Math. USSR Sbornik 70 (1991), 263-296, 297-339.

[9] Fedorov, S., Weighted Norm Inequalities and the Muckenhoupt Condition in a Multiply Connected Domain. To appear in Indiana Univ. Math. J.

[10] Fisher, S., Function Theory on Planar Domains. Whiley, 1983.

[11] Fulton, W., Algebraic Curves. Addison-Wesley, 1969.

[12] Gamelin, T. W., Uniform Algebras. Prentice-Hall, 1973.

[13] Grauert, H., Analytische Faserungen über holomorph-vollständigen Räumen. Math. Annalen 135 (1958), 263-273.

[14] Livšic, M. S., Kravitsky, N., Markus, A. S., Vinnikov, V., Theory of Commuting Nonselfadjoint Operators. Kluwer Acad. publishers, 1995.

[15] Martin, M., Putinar, M., Lectures on Hyponormal Operators. Birkhäuser, 1989. (Oper. Theory: Adv., Appl., vol. 39)

[16] McCarthy, J. E., Yang, L., Subnormal operators and quadrature domains. Advances in Math. 127 (1997), 52-72.

[17] Privalov, I. I., Boundary Behavior of Analytic Functions. German translation in: Berlin i Dentsher Verlag der Wissenchaften, 1956. MoskowLeningrad: GITTL, 1950.

[18] Solomyak, B. M., Volberg, A. L., Multiplicity of analytic Toeplitz operators. Operator Theory: Advances and Appl. 42 (1989), 87-192.

[19] Sz.-Nagy, B., Foias, C., Harmonic Analysis of Operators on a Hilbert Space. North-Holland, 1970.

[20] Treil, S., Volberg, A., Wavelets and the angle between past and future. J. Funct. Anal. 143 (1997), 269-308.

[21] Xia, D., The analytic model of a subnormal operator. Integral Eqs. Operator Theory 10 (1987), 258-289. 
[22] Xia, D., Analytic theory of subnormal operators. Integral Eqs. Operator Theory 10 (1987), 880-903.

[23] Xia, D., On pure subnormal operators with finite rank self-commutators and related operator tuples. Integral Eqs. Operator Theory 24 (1996), 106-125.

[24] Yakubovich, D. V., Riemann surface models of Toeplitz operators. $O p$ erator theory: Adv., Appl. 42 (1989), 305-415.

[25] Yakubovich, D. V., Subnormal operators of finite type I. Xia's model and real algebraic curves in $\mathbb{C}^{2}$. Revista Mat. Iberoamericana 14 (1998), 95-115.

[26] Yakubovich, D. V., Dual piecewise analytic bundle shift models of linear operators. J. Funct. Anal. 136 (1996), 294-330.

[27] Yakubovich, D. V., Dual analytic models of seminormal operators. Integral Eqs. Operator Theory 23 (1995), 353-371.

Recibido: 4 de julio de 1.997

Dmitry V. Yakubovich* Division of Mathematical Analysis Dept. of Mathematics and Mechanics St. Petersburg State University Bibliotechnaya pl. 2. St. Peterhof, St. Petersburg, 198904, RUSSIA dm@yakub.niimm.spb.su

\footnotetext{
* This research was supported, in parts, by Grant No. NW8300 from the International Science Foundation, the Mathematical Sciences Research Institute membership (September-October 1995), the Russian RFFI Grant No. 95-01-00482, Russian Federal Program "Integraciya" No. 326.53, a stipend conceded by the Interministerial Comission of Science and Technology of Spain and the grant INTAS-93-0249-EXT.
} 